\title{
A full complexity dichotomy for immanant families
}

\author{
Radu Curticapean*
}

February 9, 2021

\begin{abstract}
Given an integer $n \geq 1$ and an irreducible character $\chi_{\lambda}$ of $S_{n}$ for some partition $\lambda$ of $n$, the immanant $\operatorname{imm}_{\lambda}: \mathbb{C}^{n \times n} \rightarrow \mathbb{C}$ maps matrices $A \in \mathbb{C}^{n \times n}$ to

$$
\operatorname{imm}_{\lambda}(A)=\sum_{\pi \in S_{n}} \chi_{\lambda}(\pi) \prod_{i=1}^{n} A_{i, \pi(i)} .
$$

Important special cases include the determinant and permanent, which are the immanants associated with the sign and trivial character, respectively.

It is known that immanants can be evaluated in polynomial time for characters that are "close" to the sign character: Given a partition $\lambda$ of $n$ with $s$ parts, let $b(\lambda):=n-s$ count the boxes to the right of the first column in the Young diagram of $\lambda$. For a family of partitions $\Lambda$, let $b(\Lambda):=\max _{\lambda \in \Lambda} b(\lambda)$ and write $\operatorname{Imm}(\Lambda)$ for the problem of evaluating $\operatorname{imm}_{\lambda}(A)$ on input $A$ and $\lambda \in \Lambda$.

- If $b(\Lambda)<\infty$, then $\operatorname{Imm}(\Lambda)$ is known to be polynomial-time computable. This subsumes the case of the determinant.

- If $b(\Lambda)=\infty$, then previously known hardness results suggest that $\operatorname{Imm}(\Lambda)$ cannot be solved in polynomial time. However, these results only address certain restricted classes of families $\Lambda$.

In this paper, we show that the parameterized complexity assumption FPT $\neq \mathrm{W}[1]$ rules out polynomialtime algorithms for $\operatorname{Imm}(\Lambda)$ for any computationally reasonable family of partitions $\Lambda$ with $b(\Lambda)=\infty$. We give an analogous result in algebraic complexity under the assumption VFPT $\neq$ VW $[1]$. Furthermore, if $b(\lambda)$ even grows polynomially in $\Lambda$, we show that $\operatorname{Imm}(\Lambda)$ is hard for \#P and VNP. This concludes a series of partial results on the complexity of immanants obtained over the last 35 years.
\end{abstract}

*IT University of Copenhagen, Basic Algorithms Research Copenhagen. Supported by VILLUM Foundation grant 16582. racu@itu.dk 


\section{Introduction}

The determinant and permanent of an $n \times n$ matrix $X=\left(x_{i, j}\right)$ can be defined by the sum-product formulas

$$
\begin{aligned}
& \operatorname{det}(X)=\sum_{\pi \in S_{n}} \operatorname{sgn}(\pi) \prod_{i=1}^{n} x_{i, \pi(i)}, \\
& \operatorname{per}(X)=\sum_{\pi \in S_{n}} \prod_{i=1}^{n} x_{i, \pi(i)} .
\end{aligned}
$$

The similarity between these formulas is deceiving: While determinants admit polynomial-size circuits and can be evaluated in polynomial time, only exponential-size circuits and exponential-time algorithms are known for permanents. Valiant [37] underpinned this divide by proving that evaluating permanents is \#P-hard: Any polynomial-time algorithm for this problem would entail a polynomial-time algorithm for counting (and thus deciding the existence of) satisfying assignments to Boolean formulas, thereby collapsing P and NP. With the VNP-completeness of the permanent family [36], an analogous statement holds in algebraic complexity theory.

Unconditional lower bounds for the complexity of permanents however remain elusive, with only a quadratic lower bound on the determinantal complexity of permanents known [28, 12]. That is, expressing the permanent of an $n \times n$ matrix $X$ as the determinant of an $m \times m$ matrix (whose entries are linear forms in the entries of $X$ ) is known to require $m=\Omega\left(n^{2}\right)$. One of the core objectives in algebraic complexity theory lies in proving that $m$ must grow super-polynomially $[36,11,8]$, and this can be viewed as an algebraic version of the $\mathrm{P} \neq \mathrm{NP}$ problem.

The family of immanants. To understand the relationship between determinants and permanents better, it may help to recognize them as part of a larger family: The immanants are matrix forms that are arranged on a spectrum in which the determinant and permanent represent extreme cases. These forms were studied by Schur $[32,31]$ in the context of group character theory, and Littlewood and Richardson later explicitly introduced them as immanants [24].

Given any class function $f: S_{n} \rightarrow \mathbb{C}$, i.e., a function of permutations that depends only on the (multiset of) cycle lengths of the input permutation, the immanant $\operatorname{imm}_{f}: \mathbb{C}^{n \times n} \rightarrow \mathbb{C}$ is defined by replacing the permutation $\operatorname{sign} \operatorname{sgn}(\pi)$ in the determinant expansion with $f(\pi)$ :

$$
\operatorname{imm}_{f}(X)=\sum_{\pi \in S_{n}} f(\pi) \prod_{i=1}^{n} x_{i, \pi(i)} .
$$

In the literature, immanants are typically defined by requiring $f$ to be an irreducible character of $S_{n}$, i.e., an element from a particular basis for the vector space of class functions. ${ }^{1}$ General $f$-immanants can then be expressed as linear combinations of such immanants. Two extremal examples of irreducible characters are the trivial character $1: S_{n} \rightarrow\{1\}$ and the sign character sgn : $S_{n} \rightarrow\{-1,1\}$, which induce

$$
\begin{aligned}
& \operatorname{det}(X)=\operatorname{imm}_{\mathrm{sgn}}(X), \\
& \operatorname{per}(X)=\operatorname{imm}_{\mathbf{1}}(X) .
\end{aligned}
$$

In general, the irreducible characters of $S_{n}$ correspond naturally to partitions of $n$, as outlined in Section 3 . To see the existence of such a correspondence, note that the dimension of the space of class functions on $S_{n}$ is the number of different cycle length formats of $n$-permutations, that is, different partitions of the integer $n$. For now, let us remark that the refinement-wise minimal and maximal partitions $(1, \ldots, 1)$ and $(n)$ naturally correspond to the sign and trivial character, respectively. As another example, we have

$$
\chi_{(2,1, \ldots, 1)}(\pi)=\operatorname{sgn}(\pi) \cdot(\#\{\text { fixed points of } \pi\}-1) .
$$

\footnotetext{
${ }^{1}$ The resulting immanants are sometimes also called character immanants, as opposed to other types of immanants, such as the Kazhdan-Lusztig immanants [29].
} 
Abbreviating $\operatorname{imm}_{\lambda}=\operatorname{imm}_{\chi_{\lambda}}$, we have det $=\operatorname{imm}_{(1, \ldots, 1)}$ and per $=\operatorname{imm}_{(n)}$. Likewise, $\operatorname{imm}_{(2,1, \ldots, 1)}$ sums over row-column permutations of a matrix with weights as given in (1). For a more applied example, it is known that the number of Hamiltonian cycles in a directed $n$-vertex graph $G$, i.e., the immanant associated with the indicator function for cyclic permutations (evaluated on the adjacency matrix of $G$ ) can be written as a linear combination of the hook immanants $\operatorname{imm}_{\left(r, 1^{n-r}\right)}$ for $1 \leq r \leq n$.

Beyond their theoretical origins in group character theory, immanants have been applied in combinatorial chemistry [13] and linear optics [34], and they feature in (conjectured) inequalities in matrix analysis [33]. In this paper, we focus on complexity-theoretic aspects of immanants and their role as an interpolating family between determinants and permanents.

The complexity of immanants. It is known that irreducible characters of $S_{n}$ can be evaluated in polynomial time $[9,8]$. Using this, any character immanant of an $n \times n$ matrix can be evaluated in $n ! \cdot n^{O(1)}$ time by brute-force, or in $2^{n+o(n)}$ time by a variant of the Bellman-Held-Karp dynamic programming approach for Hamiltonian cycles. For some immanants however, among them the determinant, this exponential running time is far from optimal: Hartmann [23] gave an algorithm for evaluating $\operatorname{imm}_{\lambda}$ in $O\left(n^{6 b(\lambda)+4}\right)$ time, where $b(\lambda):=n-s$ for a partition $\lambda=\left(\lambda_{1}, \ldots, \lambda_{s}\right)$ with $s$ parts. In visual terms, the quantity $b(\lambda)$ counts the boxes to the right of the first column in the Young diagram of $\lambda$, which is a left-aligned shape whose $i$-th row contains $\lambda_{i}$ boxes, when $\lambda$ is ordered non-increasingly:

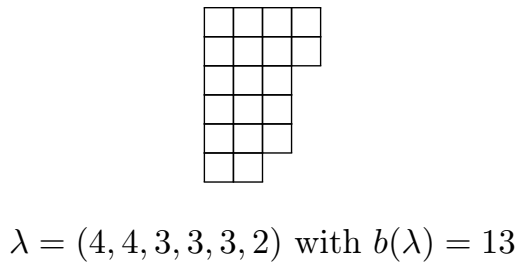

In particular, partitions $\lambda$ with $b(\lambda)=O(1)$ induce polynomial-time solvable immanants. Barvinok [1] and Bürgisser [10] later gave algorithms with improved running times $O\left(n^{2} d_{\lambda}^{4}\right)$ and $O\left(n^{2} s_{\lambda} d_{\lambda}\right)$, where $s_{\lambda}$ and $d_{\lambda}$ denote the numbers of standard and semi-standard tableaux of shape $\lambda .^{2}$ These algorithms give better running times in the exponential-time regime, but they do not identify new polynomial-time solvable immanants. One is therefore naturally led to wonder whether $b(\lambda)$ is indeed the determining parameter for the complexity of immanants. To investigate this formally, we consider families of partitions $\Lambda$ and define $\operatorname{Imm}(\Lambda)$ as the problem of evaluating $\operatorname{imm}_{\lambda}(A)$ on input a matrix $A$ and a partition $\lambda \in \Lambda$. As discussed above, the problem $\operatorname{Imm}(\Lambda)$ is polynomial-time solvable if the quantity

$$
b(\Lambda):=\max _{\lambda \in \Lambda} b(\lambda)
$$

is finite. On the other hand, for various families $\Lambda$ with unbounded $b(\Lambda)$, the problem $\operatorname{Imm}(\Lambda)$ is indeed known to be hard for the counting complexity class \#P and its algebraic analog VNP:

- Bürgisser [9] showed VNP-completeness and \#P-hardness of $\operatorname{Imm}(\Lambda)$ for any family $\Lambda$ of hook partitions $\left(t(n), 1^{n-t(n)}\right)$, provided that $t=\Omega\left(n^{\alpha}\right)$ with $\alpha>0$ can be computed in polynomial time. A similar result appears in Hartmann's work [23].

- In the same paper, Bürgisser showed similar hardness results for families of rectangular partitions of polynomial width. (The width is the largest entry in the partition.)

In his 2000 monograph [8], Bürgisser conjectures that $\operatorname{Imm}(\Lambda)$ is hard for any reasonable family $\Lambda$ of polynomial width. He also asks about the complexity status of partitions of width 2 , and overall deems the complexity of immanants to be "still full of mysteries". Some of these mysteries have since been resolved:

\footnotetext{
${ }^{2}$ Given a partition $\lambda$ of $n$, a standard tableau of shape $\lambda$ is an assignment of the numbers $1, \ldots, n$ to the boxes in the Young diagram of $\lambda$ such that all rows and columns are strictly increasing. In a semi-standard tableau, rows are only required to be non-decreasing.
} 
- In 2003, Brylinski and Brylinski [7] showed VNP-completeness for any family of partitions $\Lambda$ with a gap of width $\Omega\left(n^{\alpha}\right)$ for $\alpha>0$. Here, a gap is the difference between two consecutive rows.

- In 2013, Mertens and Moore [27] proved \#P-hardness for the family $\Lambda$ of all partitions of width 2, that is, the partitions containing only entries 1 and 2 . They also proved $\oplus \mathrm{P}$-hardness for the more restricted family of partitions containing only the entry 2 .

- In the same year, de Rugy-Altherre [20] gave a dichotomy for partition families $\Lambda$ of constant width and polynomial growth of $b(\lambda)$, confirming for such families that boundedness of $b(\Lambda)$ indeed determines the complexity of $\operatorname{Imm}(\Lambda)$.

However, an exhaustive complexity classification of $\operatorname{Imm}(\Lambda)$ for general partition families $\Lambda$ still remained open, even 35 years after Hartmann's initial paper [23] and despite several appearances as an open problem [27, 20], also in a monograph [8]. In fact, even very special cases like $\operatorname{Imm}(\Lambda)$ for the staircase partitions $(k, k-1, \ldots, 1)$ remained unresolved [20].

\subsection{Our results}

We classify the complexity of the problems $\operatorname{Imm}(\Lambda)$ for partition families $\Lambda$ satisfying natural computability and density conditions that are satisfied by all families studied in the literature. Under the assumption $\mathrm{FPT} \neq \# \mathrm{~W}[1]$ from parameterized complexity [21], we confirm that $\operatorname{Imm}(\Lambda)$ is polynomial-time solvable iff $b(\Lambda)$ is unbounded. An algebraic analogue holds under the assumption VFPT $\neq \mathrm{VW}[1]$ introduced by Bläser and Engels [6]. (Please consider Section 2.2 for a brief introduction to the relevant complexity classes.) Our classification holds even if $b(\lambda)$ only grows sub-polynomially in $\Lambda$, which allows us to address families such as

$$
\Lambda_{\log }=\left\{\left(\lceil\log n\rceil, 1^{n}\right) \mid n \in \mathbb{N}\right\} .
$$

Note that $\operatorname{Imm}\left(\Lambda_{\log }\right)$ can be solved in $n^{O(\log n)}$ time by the $n^{O(b(\lambda))}$ time algorithms discussed before, which likely prevents hardness for \#P or VNP. At the same time, a polynomial-time algorithm seems unlikely. Thus, partition families like $\operatorname{Imm}\left(\Lambda_{\mathrm{log}}\right)$ fall into the "blind spot" of classical dichotomies, an issue that is also alluded to in [20].

Our sanity requirements on $\Lambda$ are encapsulated as follows: We say that $\Lambda$ supports growth $g: \mathbb{N} \rightarrow \mathbb{N}$ if every $n \in \mathbb{N}$ admits a partition $\lambda^{(n)} \in \Lambda$ with $b\left(\lambda^{(n)}\right) \geq g(n)$ and total size $\Theta(n)$. This ensures that $\Lambda$ is dense enough and that $\Lambda$ supplies sufficiently many boxes both in the first column and to the right of it. We may also require that $\lambda^{(n)}$ can be computed in polynomial time on input $n \in \mathbb{N}$ and then say that $\Lambda$ computationally supports growth $g$. This condition is not required for the algebraic completeness results.

Example. The family of staircase partitions $(n, n-1, \ldots, 1)$ supports growth $\Omega(n)$. The partition families $\left(\lceil\log n\rceil, 1^{n}\right)$ and $\left(n, 1^{2^{n}}\right)$ for $n \in \mathbb{N}$ support growth $\Omega(\log n)$, even though the second family is exponentially sparse. On the other hand, partition families whose sizes grow doubly exponentially do not support any growth by our definition. It might still be possible to address such families via "infinitely often" versions of \#P or VNP, but we currently see no added value in doing so.

In the polynomial growth regime for $b(\lambda)$, we obtain classical \#P-hardness and VNP-completeness results. As a bonus, we also obtain the expected quantitative lower bounds under the exponential-time hypothesis \#ETH, which postulates that counting satisfying assignments to $n$-variable 3 -CNFs takes $\exp (\Omega(n))$ time.

Theorem 1. For any family of partitions $\Lambda$ :

- If $b(\Lambda)<\infty$, then $\operatorname{Imm}(\Lambda) \in \mathrm{FP}$ and $\operatorname{Imm}(\Lambda) \in \mathrm{VP}$.

- Otherwise, if $\Lambda$ supports growth $\Omega\left(n^{\alpha}\right)$ for some $\alpha>0$, then $\operatorname{Imm}(\Lambda)$ is VNP-complete. If $\Lambda$ computationally supports growth $\Omega\left(n^{\alpha}\right)$, then $\operatorname{Imm}(\Lambda)$ is \#P-hard and admits no $\exp \left(o\left(n^{\alpha}\right)\right)$ time algorithm unless \#ETH fails. 
Theorem 1 subsumes all known VNP-hardness and \#P-hardness results for immanant families, confirms the conjecture from [27], and settles the case of staircases. Using parameterized complexity theory, we also address the sub-polynomial growth regime for $b(\lambda)$. To this end, we consider parameterized problems, whose instances $(x, k)$ come with a parameter $k$. The corresponding objects in the algebraic setting are parameterized polynomial families $\left(p_{n, k}\right)$, where the second index $k$ is a parameter. A parameterized problem (or polynomial family) is said to be fixed-parameter tractable if it can be solved in $f(k) \cdot n^{O(1)}$ time with $n=|x|$ (or admits circuits of that size) for some computable function $f$. The problem (or polynomial family) is then said to be contained in FPT (or VFPT). The classes \#W[1] $\supseteq$ FPT (and VW[1] $\supseteq$ VFPT) contain problems (and polynomial families) that are believed not to be fixed-parameter tractable.

Theorem 2. For any family of partitions $\Lambda$ :

- If $b(\Lambda)<\infty$, then $\operatorname{Imm}(\Lambda) \in \mathrm{FP}$ and $\operatorname{Imm}(\Lambda) \in \mathrm{VP}$.

- Otherwise, if $\Lambda$ supports growth $g \in \omega(1)$, then $\operatorname{Imm}(\Lambda) \notin \mathrm{VP}$ unless $\mathrm{VFPT}=\mathrm{VW}[1]$. If $\Lambda$ computationally supports growth $g$, then $\operatorname{Imm}(\Lambda) \notin \mathrm{FP}$ unless $\mathrm{FPT}=\# \mathrm{~W}[1]$.

Note that we do not prove $\operatorname{Imm}(\Lambda)$ to be hard for \#W[1] or VW[1] as a parameterized problem with parameter $b(\lambda)$, even though this might seem natural. Indeed, problems like $\operatorname{Imm}\left(\Lambda_{\log }\right)$ are trivially fixedparameter tractable in the parameter $b(\lambda)$. We only show that polynomial-time algorithms for $\operatorname{Imm}(\Lambda)$ would render \#W[1]-hard problems fixed-parameter tractable.

\subsection{Proof outline}

We establish Theorems 1 and 2 by reduction from \#Match, the problem of counting $k$-matchings in bipartite graphs $H$. When parameterized by $k$, this problem is \#W[1]-complete $[4,15,19,18]$, with an analogous statement in the algebraic setting [6]. When $k$ grows polynomially in $|V(H)|$, counting $k$-matchings is complete for \#P and VNP by a trivial reduction from the permanent [37].

To reduce counting matchings to immanants, we proceed in three stages: First, we identify two types of "exploitable resources" in partitions, then we show how to exploit them for a reduction, and finally we wrap the proof up in complexity-theoretic terms.

\section{Extracting resources (Section 4)}

Our construction relies on two types of resources that can supplied by a given partition: A large staircase or a large number of non-vanishing tetrominos.
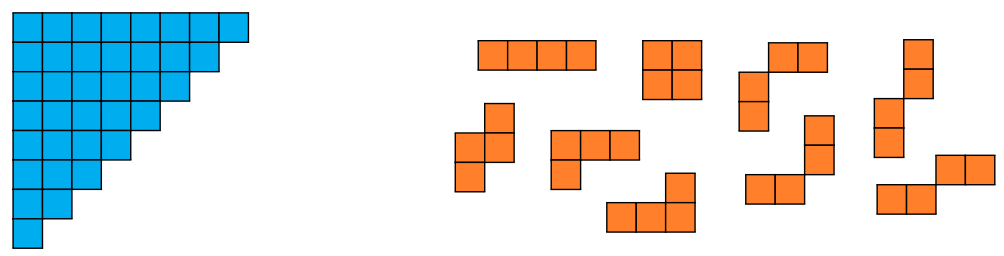

To define these notions, consider successively "peeling" dominos $\square$ and $\boxminus$ from $\lambda$, that is, removing them from the south-eastern border of $\lambda$ while ensuring that the shape obtained after each step has non-increasing row lengths. After peeling the maximum number of dominos this way, we reach some (possibly empty) staircase $\mu$, which is easily seen to be unique. The domino number $d(\lambda)$ is this maximum number of removable dominos, and $\mu$ is the staircase of $\lambda$; we write $w(\lambda)$ for its width.

Now consider peeling two dominos from a partition $\lambda$. Some of the shapes that can arise this way are shown above in orange; we call them "non-vanishing tetrominos" for reasons that will become evident in the proof. For the four corner-connected domino pairs, we adopt the convention that their two dominos must be peeled successively from disjoint rows and columns of $\lambda$. The non-vanishing tetromino number $s(\lambda)$ then is 
the maximum number of non-vanishing tetrominos that can be peeled from $\lambda$. Note that this number is 0 for the determinant-inducing partition $(1, \ldots, 1)$ and $\lfloor n / 4\rfloor$ for the permanent-inducing partition $(n)$.

In Section 4, we establish a "win-win situation" for these resources: For any partition $\lambda$, at least one of $w(\lambda) \in \Omega(\sqrt{b(\lambda)})$ or $s(\lambda) \in \Omega(b(\lambda))$ must hold.

\section{Exploiting resources (Sections 5 and 6)}

Next, we outline how to exploit staircases and non-vanishing tetrominos in a partition $\lambda$ for reductions from counting $k$-matchings to evaluating $\mathrm{imm}_{\lambda}$. Throughout this paper, the immanant of a directed graph $G$ refers to the immanant of its adjacency matrix $A$. This way, we can view immanants as character-weighted sums over the cycle covers of digraphs.

Given an $n$-vertex graph $H$ and $k \in \mathbb{N}$, we construct a digraph $G$ such that $\operatorname{imm}_{\lambda}(G)$ counts the $k$ matchings in $H$ up to a constant factor $c_{\lambda, k}$ that can be computed in polynomial time. In a second step, we show that the constant factor $c_{\lambda, k}$ is non-zero if $\lambda$ supplies enough resources. Both the construction of $G$ and the handling of $c_{\lambda, k}$ differ for staircases and tetrominos, as outlined below.

Non-vanishing tetrominos (Section 6) Each non-vanishing tetromino peeled from $\lambda$ enables a particular edge gadget: To count the $k$-matchings in a graph $H$, we replace each edge $u v \in E(H)$ by the gadget shown below; the weight $w$ of $u v$ appears on two edges of the gadget. Together with additional constructions detailed in Section 6, this results in a directed graph $G$.

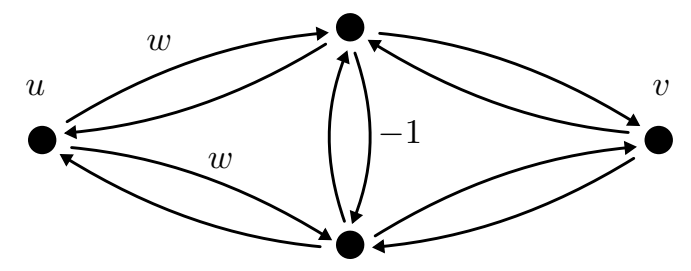

The edge gadget effectively constrains the set of cycle covers counted by the immanant, as undesired cycle covers cancel out in pairs of opposite signs. In the remaining cycle covers of $G$, each gadget is either in the passive state (shown below in cyan) or in one of four active states (two are shown below in green, two more are symmetric versions thereof).
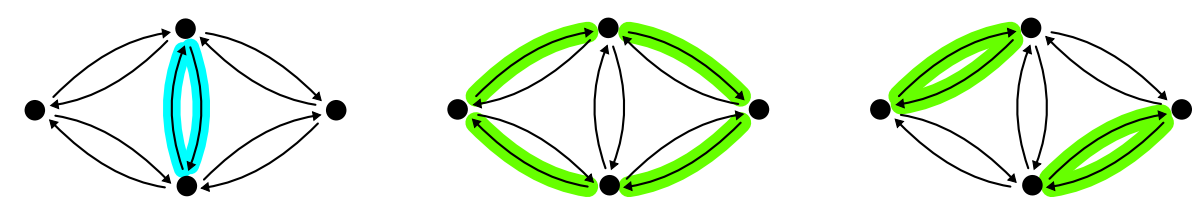

This allows us to simulate matchings $M$ in $H$ via cycle covers in $G$ : We interpret active gadgets as matching edges $e \in M$ and passive gadgets as edges $e \in E(H) \backslash M$. Intuitively speaking, each active gadget "uses up" one non-vanishing tetromino of $\lambda$, while passive gadgets only require a domino. Overall, if we can peel $O(k)$ non-vanishing tetrominos and some number of dominos from $\lambda$, then $\operatorname{imm}_{\lambda}$ can be used to count $k$-matchings in $H$.

Large staircase (Section 5) If the staircase $\mu$ of $\lambda$ contains $\Omega(k)$ boxes, then properties of the staircase character $\chi_{\mu}$ enable an ad-hoc reduction from counting $k$-matchings in bipartite graphs to the $\lambda$-immanant. More specifically, we observe and use that cycle covers with even cycles vanish in staircase characters $\chi_{\mu}$. After discarding irrelevant dominos from $\lambda$, we can then use this fact together with a particular graph construction to compute a sum over cycle covers with one particular fixed cycle length format by reduction to $\operatorname{imm}_{\lambda}$. This way of exploiting staircase characters to avoid even-length cycles may also be useful for other algorithmic applications. 


\section{Wrap-up (Section 7)}

For a streamlined presentation, the two reductions above are encapsulated as mere mathematical formulas relating the number of $k$-matchings in a graph $H$ with the immanant of a digraph $G$ constructed from $H$. In Section 7, we add the necessary "wrapper code" to obtain the (parameterized and polynomial-time, algebraic and computational) reductions required to prove Theorems 1 and 2.

\subsection{Proof highlights}

Most of our arguments rely on making non-vanishing tetrominos and staircases come together in just the right way. Additionally, the following can be pointed out:

- In the tetromino-based reduction, the particular form of active and passive states in edge gadgets ensures that we only need to understand character values $\chi_{\lambda}(\rho)$ on cycle formats $\rho$ with cycle lengths 1 , 2 , and 4. This allows us to sidestep more involved representation-theoretic arguments that occur in related works. It should be noted that equality and exclusive-or gadgets that enforce consistency properties of cycle covers are common in algebraic and counting complexity, dating back to Valiant [36, 37]. The particular idea of repurposing an equality gadget into an edge gadget was also already used in the author's very first paper with Bläser [3].

- Parameterized complexity assumptions allow us to handle cases that cannot be addressed in classical frameworks, such as the family $\operatorname{Imm}\left(\Lambda_{\log }\right)$ discussed before. By basing hardness on the assumptions $\mathrm{FPT} \neq \# \mathrm{~W}[1]$ and VFPT $\neq \mathrm{VW}[1]$, we can still argue about such families.

- Two inconspicuous but crucial proof steps (Lemmas 22 and Fact 29) rely on cute arguments involving "dominos on chessboards" that one would rather expect in the context of recreational mathematics. This can again be credited to the edge gadget, as such arguments would likely fail for cycle lengths other than 2 and 4 .

\section{Preliminaries}

We start with basic definitions for graphs and partitions in Section 2.1. In Sections 2.2 and 2.3, we present the complexity-theoretic preliminaries used in this paper. A bare-bones introduction to the relevant character theory of symmetric groups can be found in Section 3.

\subsection{Basic notions}

Graphs. We consider undirected graphs (when counting matchings) and directed graphs (when evaluating immanants). Both graph types may feature (indeterminate) edge-weights, and directed graphs may feature self-loops. For a graph $G$ with adjacency matrix $A$, we write $\operatorname{imm}(G)$ instead of $\operatorname{imm}(A)$ and view the immanant as a sum over cycle covers: A partial cycle cover in a directed graph $G$ is an edge-set $C \subseteq E(G)$ such that each vertex has one incoming and one outgoing edge in $C$. It is a cycle cover if all of $V(G)$ is covered. Directed 2-cycles will also be called digons.

Partitions. A partition of a positive integer $n \in \mathbb{N}$ is a multi-set $\lambda$ of positive integers such that $\sum_{i \in \lambda} i=n$. Its elements are called parts, and we write $\lambda \vdash n$ to indicate that $\lambda$ is a partition of $n$. Several notations will be used for partitions:

sorted tuple

$(4,4,3,3,3,2)$ compact notation

$\left(4^{2}, 3^{3}, 2^{1}\right)$
Young diagram

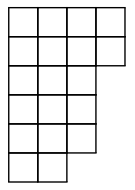


As depicted above, the Young diagram of a partition $\lambda=\left(\lambda_{1}, \ldots, \lambda_{s}\right)$ is a left-aligned shape consisting of $\lambda_{i}$ boxes in row $i$. We define the gap $\delta_{i}$ of row $i$ as $\delta_{i}:=\lambda_{i}-\lambda_{i+1}$, where we consider $\lambda_{s+1}:=0$.

Given partitions $\lambda \vdash n$ and $\lambda^{\prime} \vdash n^{\prime}$, we sometimes abuse notation and write $\left(\lambda, \lambda^{\prime}\right)$ for the partition $\lambda \cup \lambda^{\prime}$ of $n+n^{\prime}$. Sometimes we also specify an ordering for the elements in a partition: An ordered partition of $n$ (also called composition) is a tuple of positive integers that sum to $n$. We will state explicitly when partitions are considered to be ordered.

Let us stress that any permutation $\pi$ of $n$ elements (and any cycle cover $C$ of an $n$-vertex graph) naturally induces a partition $\lambda \vdash n$ through its cycle lengths, the cycle format $\rho(\pi)$ of $\pi$. For example, the identity permutation has cycle format $(1, \ldots, 1)$ and cyclic permutations have cycle format $(n)$. We also say that a cycle cover is a $\rho$-cycle cover if its format is $\rho$.

\subsection{Complexity-theoretic notions}

We recall basic notions from complexity theory. For a more comprehensive overview, please consider [37, 21, 17] for (parameterized) counting complexity and $[36,6,11,8]$ for (parameterized) algebraic complexity.

Counting complexity. We view functions $f:\{0,1\}^{*} \rightarrow \mathbb{Q}$ as counting problems. For example, the problem \#SAT maps (binary encodings of) Boolean formulas $\varphi$ to the number of satisfying assignments in $\varphi$. Properly encoded, the permanent of rational-valued matrices is a counting problem. A counting problem is contained in FP if it can be solved in polynomial time.

A polynomial-time Turing reduction from a counting problem \#A to another counting problem \#B is a polynomial-time algorithm that solves \#A with an oracle for \#B. We say that \#B is \#P-hard (under Turing reductions) if \#SAT admits a polynomial-time Turing reduction to \#B. While more stringent reduction notions exist, they are not relevant for the purposes of this paper. Assuming FP $\neq$ \#P, no \#P-hard problem can be solved in polynomial time.

A parameterized counting problem features inputs $(x, k)$ for $x \in\{0,1\}^{*}$ and $k \in \mathbb{N}$. It is fixedparameter tractable if it can be solved in time $f(k) \cdot|x|^{O(1)}$ for some computable function $f$, and we write FPT for the class of such problems. A parameterized Turing reduction from a parameterized counting problem \#A to another parameterized problem \#B is an algorithm that solves any instance $(x, k)$ for \#A in time $f(k) \cdot|x|^{O(1)}$ with an oracle for \#B that is only called on instances $\left(y, k^{\prime}\right)$ with $k^{\prime} \leq g(k)$. Here, both $f$ and $g$ are computable functions. We say that \#B is \#W[1]-hard if the problem of counting $k$-cliques in a graph admits a parameterized Turing reduction to \#B. Assuming FPT $\neq \# \mathrm{~W}[1]$, no \#W[1]-hard problem is fixed-parameter tractable.

The (counting version of the) exponential-time hypothesis \#ETH postulates that no $\exp (o(n))$ time algorithm solves \#SAT on $n$-variable formulas. If a problem \#A cannot be solved in $\exp (o(n))$ time, and it admits a polynomial-time Turing reduction to a problem \#B such that every invoked oracle query has size $O\left(n^{c}\right)$ for $c \geq 0$, then \# B cannot be solved in $\exp \left(o\left(n^{1 / c}\right)\right)$ time. Likewise, if a parameterized problem \#A cannot be solved in $f(k) \cdot n^{o(k)}$ time and it admits a parameterized Turing reduction to a problem \#B such that every invoked query $\left(y, k^{\prime}\right)$ satisfies $k^{\prime} \leq O(k)$, then \#B also cannot be solved in $f(k) \cdot n^{o(k)}$ time. The hypothesis \#ETH rules out an $f(k) \cdot n^{o(k)}$ time algorithm for counting $k$-cliques and thus implies $\mathrm{FPT} \neq \# \mathrm{~W}[1]$.

Algebraic complexity. In the algebraic setting, p-families play the role of counting problems: A sequence of multivariate polynomials $f=\left(f_{1}, f_{2}, \ldots\right)$ over some field is a p-family if, for all $n \in \mathbb{N}$, the degree and the number of variables of $f_{n}$ are bounded by $n^{O(1)}$. For example, the sequences of determinants and permanents of $n \times n$ matrices with indeterminates are p-families. A p-family is contained in VP if it admits a polynomial-size arithmetic circuit.

A p-family $f$ admits a c-reduction to another p-family $g$ if there is an arithmetic circuit of polynomial size that computes each $f_{n}$ with oracle gates for $g_{1}, \ldots g_{n} O(1)$. A p-family $g$ is VNP-hard if the permanent family admits a c-reduction to $g$. Assuming VP $\neq$ VNP, no VNP-hard family is contained in VP. 
A parameterized p-family is a family $f=\left(f_{n, k}\right)_{n, k \in \mathbb{N}}$ with two indices such that $f_{n, k}$ uses $n^{O(1)}$ variables and has degree $(n+k)^{O(1)}$. A family $f$ is contained in VFPT if it admits an arithmetic circuit of size $h(k) \cdot n^{O(1)}$ for some function $h$. An example for a parameterized p-family is given by the partial permanents $\operatorname{per}_{n, k}=\sum_{\pi \in S(n, k)} \prod_{i=1}^{n} x_{i, \pi(i)}$ for $n, k \in \mathbb{N}$, where $S(n, k)$ is the set of all permutations with $n-k$ fixed points. A parameterized family $f=\left(f_{n, k}\right)_{n, k \in \mathbb{N}}$ admits a parameterized c-reduction to another family $g=\left(g_{n, k}\right)_{n, k \in \mathbb{N}}$ if there is an arithmetic circuit of size $h(k) \cdot n^{O(1)}$ that computes $f_{n, k}$ with oracle gates for polynomials $g_{n^{\prime}, k^{\prime}}$ satisfying $n^{\prime} \leq h(k) \cdot n^{O(1)}$ and $k^{\prime} \leq h(k)$ for some function $h$. We say that $g$ is VW[1]-hard if the partial permanents admit a parameterized c-reduction to $g$. Assuming VFPT $\neq$ VW[1], no VW[1]-hard problem is contained in VFPT.

\subsection{Counting matchings}

A matching in an undirected graph $H$ is a set $M \subseteq E(H)$ of pairwise disjoint edges. We write $\mathcal{M}_{k}(H)$ for the set of matchings with $k$ edges in $H$. A matching $M$ is a perfect matching if every vertex $v \in V(H)$ is contained in some edge of $M$. Given an $n$-vertex graph $H$ with edge-weights $w: E(H) \rightarrow \mathbb{Q}$, we define

$$
\begin{aligned}
\# \operatorname{Match}(H, k) & =\sum_{M \in \mathcal{M}_{k}(H)} \prod_{e \in M} w(e), \\
\# \operatorname{PerfMatch}(H) & =\# \operatorname{Match}(H,|V(H)| / 2) .
\end{aligned}
$$

Note that \#PerfMatch $(H)$ is only defined for graphs $H$ with an even number of vertices. We prove hardness of immanants by reduction from (restrictions of) the problem of evaluating \#Match on bipartite graphs.

Definition 3. The counting problem \#PerfMatch asks to compute \#PerfMatch $(H)$ for bipartite graphs $H$ with edge-weights $w: E(H) \rightarrow \mathbb{Q}$.

For any fixed polynomial-time computable function $g: \mathbb{N} \rightarrow \mathbb{N}$, we define the counting problem \#Match ${ }^{(g)}$ : Given a pair $(H, k)$ consisting of an undirected bipartite graph $H$ with edge-weights $w: E(H) \rightarrow \mathbb{Q}$ and a number $k \leq g(|V(H)|)$, compute \#Match $(H, k)$.

On the complete bipartite graphs $K_{n, n}$ with indeterminate edge-weights, \#PerfMatch induces the p-family of permanent polynomials, and \# Match ${ }^{(g)}$ likewise induces a restriction of the partial permanent family with \#Match ${ }_{n, k}^{(g)}=0$ for $k>g(n)$. Abusing notation, we call these p-families \#PerfMatch and \#Match ${ }^{(g)}$ as well. Note that no graphs are given as inputs to these families; the numbers of $k$-matchings for given bipartite graphs $H$ can be obtained by evaluating the polynomials at points whose non-zero coordinates encode the edges of $H$.

The hardness results for \#PerfMatch and \#Match ${ }^{(g)}$ required in the remainder of the paper are either known in the literature or can be derived easily. We collect the relevant results below.

Theorem 4. The following holds:

1. The problem \#PerfMatch is VNP-complete and \#P-hard and admits no $2^{o(n)}$ time algorithm under \#ETH, even on bipartite graphs of maximum degree 3.

2. For any unbounded and polynomial-time computable function $g$, the problem \# $\mathrm{Match}^{(g)}$ is \#W[1]-hard and $\mathrm{VW}[1]$-complete.

Proof. See $[37,36]$ for the \#P-hardness and VNP-completeness of \#PerfMatch and [16] for the lower bound under \#ETH. The \#W[1]-hardness of the cardinality-unrestricted problem \#Match is shown in [19], and and VW[1]-completeness is shown in [6, Lemma 8.7].

For any polynomial-time computable function $g$, we give a parameterized reduction from \#Match to \# $\operatorname{Match}^{(g)}$ : Any instance $(H, k)$ with an $n$-vertex graph and $k \leq g(n)$ can be solved directly with a call to \#Match ${ }^{(g)}$. On the other hand, if $k>g(n)$, then we have $n<g^{-1}(k)$, so we can count $k$-matchings in $H$ by brute-force in $g^{\prime}(k)$ time for some computable function $g^{\prime}$. This satisfies the requirements of a parameterized 
reduction. In the algebraic setting, we need not branch on $g(n)$ within a circuit, but instead hard-code into the circuit family realizing \#Match whether to (i) perform a brute-force sum over matchings, or (ii) use the oracle gate for $\# \operatorname{Match}^{(g)}$.

\section{Characters of the symmetric group}

We give a minimal introduction to representations and characters of the symmetric group; this material is covered thoroughly in classical textbooks [22, 26, 30,25]. For our purposes, a representation of $S_{n}$ is a homomorphism $f$ from $S_{n}$ to the group $\mathrm{GL}_{t}(\mathbb{C})$ of invertible $t \times t$ matrices, for some dimension $t \in \mathbb{N}$. Examples include the trivial representation that maps all of $S_{n}$ to 1 , the sign representation that maps permutations to their sign, and the permutation matrix representation that maps each permutation to its $n \times n$ permutation matrix.

A representation $f: S_{n} \rightarrow \mathrm{GL}_{t}(\mathbb{C})$ is irreducible if no proper subspace of $\mathbb{C}^{t}$ is invariant under all the transformations $f(\pi)$ for $\pi \in S_{n}$. Among the examples given before, this holds trivially for the trivial and sign representations. The permutation matrix representation however is not irreducible for $n>1$, as every permutation matrix maps the 1 -dimensional subspace of $\mathbb{C}^{n}$ spanned by $(1, \ldots, 1)$ to itself.

\subsection{Characters}

Characters condense essential information about representations $f: S_{n} \rightarrow \mathrm{GL}_{t}(\mathbb{C})$ into scalar-valued functions $\chi_{f}: S_{n} \rightarrow \mathbb{C}$. For us, they play the role of "generalized signs" in the sum-product definition of immanants.

Definition 5. The character of a representation $f$ is the function $\chi_{f}: S_{n} \rightarrow \mathbb{C}$ that maps $\pi \in S_{n}$ to the trace of the matrix $f(\pi)$.

The trivial and sign representations coincide trivially with their characters. The character of the permutation matrix representation counts the fixed points of a permutation.

Characters of representations are class functions, which are functions $f: S_{n} \rightarrow \mathbb{C}$ that depend only on the cycle format of the input. These functions form a vector space by point-wise linear combinations, and a particularly useful basis for this space is given by the irreducible characters, which are the characters of irreducible representations. The set of irreducible characters corresponds bijectively to the partitions of $n$.

For $S_{2}$, the only irreducible characters are the trivial character $\chi_{(2)}$ and sign character $\chi_{(1,1)}$. There are five irreducible characters for $S_{4}$; their values $\chi_{\lambda}(\rho)$ are shown below as a character table.

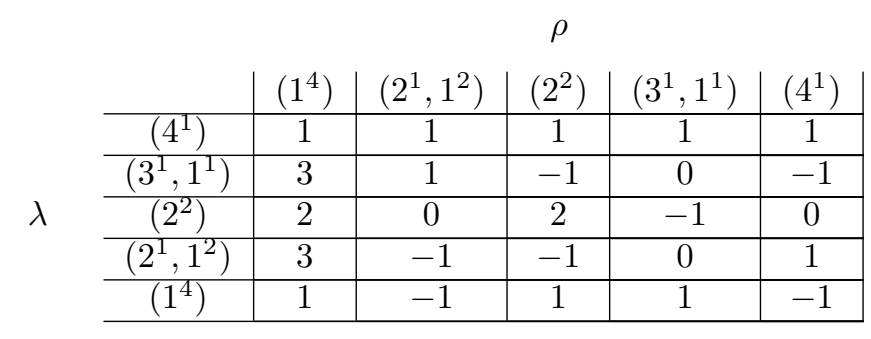

In the next subsections, we describe the Murnaghan-Nakayama rule, a combinatorial method for calculating character values $\chi_{\lambda}(\rho)$, together with a simple extension thereof that applies to particular linear combinations of character values. To state these rules, we need to introduce several types of tableaux.

\subsection{Skew shapes and their tableaux}

Recall that partitions $\lambda$ can be described by Young diagrams, such as

Given such a diagram, a tableau is obtained by writing numbers (or other objects) into the boxes, subject to some specified rules. The representation theory of $S_{n}$ abounds in different types of tableaux - we introduce yet another such type, the skew shape tableaux. 


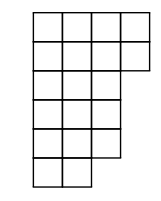

$\left(4^{2}, 3^{3}, 2^{1}\right)$.

Definition 6. Let $\lambda, \mu$ be partitions such that $\mu_{i} \leq \lambda_{i}$ for all rows $i$ of $\lambda$. We say that $\mu$ is contained in $\lambda$ and define the skew shape $\lambda / \mu$ by removing the diagram of $\mu$ from $\lambda$.

Consider the two examples below. The right example shows that skew shapes need not be connected; we call a skew shape connected if each pair of boxes can be reached by a path in the interior of the shape.

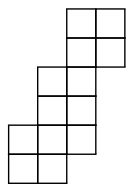

$\left(4^{2}, 3^{3}, 2^{1}\right) /\left(2^{2}, 1^{2}\right)$

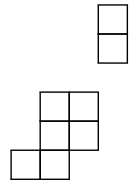

$\left(4^{2}, 3^{3}, 2^{1}\right) /\left(3^{3}, 1^{2}\right)$

Table 1 lists the connected skew shapes on 4 boxes. Any general skew shape on $b$ boxes is obtained by choosing connected skew shapes with a total of $b$ boxes and arranging them in disjoint rows and columns.

We will often peel skew shapes $\gamma$ from other skew shapes $\lambda / \mu$. Our definition of this process allows for peeling different components of $\gamma$ from different places.

Definition 7. A skew shape $\gamma$ can be peeled from $\lambda / \mu$ if there is a partition $\lambda^{\prime}$ contained between $\mu$ and $\lambda$ such that $\lambda / \lambda^{\prime}$ equals $\gamma$ after deleting empty rows and columns from both $\lambda / \lambda^{\prime}$ and $\gamma$.

A skew shape tableau is obtained by successively peeling skew shapes from $\lambda$.

Definition 8. Let $\tilde{\Gamma}=\left(\Gamma_{1}, \ldots, \Gamma_{s}\right)$ be such that $\Gamma_{i}$ for $i \in[s]$ is a set of skew shapes on the same number $n_{i}$ of boxes. Let $\lambda$ be a partition of $n=\sum_{i} n_{i}$. A skew shape tableau of $\lambda$ with format $\tilde{\Gamma}$ is obtained by successively peeling skew shapes $\gamma_{1}, \ldots, \gamma_{s}$ with $\gamma_{i} \in \Gamma_{i}$ from $\lambda$ and labeling $\gamma_{i}$ with $i$ in $\lambda$. We write $\mathcal{S}(\lambda, \tilde{\Gamma})$ for the set of such tableaux.

Three skew shape tableaux with different formats are shown below. The boxes are colored rather than numbered. The third tableau is even a border strip tableau, as defined in the next subsection.

\subsection{An extension of the Murnaghan-Nakayama rule}

The Murnaghan-Nakayama rule expresses the character value $\chi_{\lambda}(\rho)$ for partitions $\lambda$ and $\rho$ as a signed sum over particular skew shape tableaux of $\lambda$. The sign of a tableau is determined by the parity of odd-height shapes. In our later arguments, $\rho$ will always be the format of a cycle cover.

Definition 9. A border strip is a connected skew shape not containing any $2 \times 2$ square. A border strip tableau of $\lambda \vdash n$ is a skew shape tableau consisting only of border strips. Given a partition $\lambda$ and an ordered partition $\kappa$, we write $\mathcal{B}(\lambda, \kappa)$ for the set of border strip tableaux of $\lambda$ in which the $i$-th shape has $\kappa_{i}$ boxes.

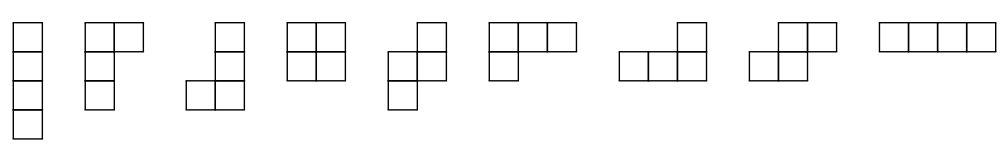

Table 1: All connected skew shapes on 4 boxes. 

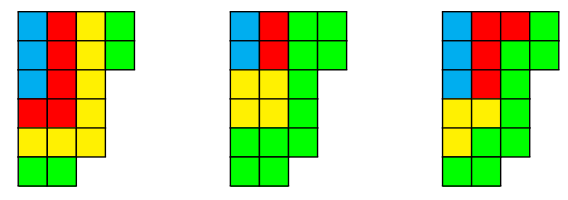

The height $\operatorname{ht}(\gamma)$ of a border strip $\gamma$ is the number of occupied rows in $\gamma$ minus 1. Given a skew shape tableau $T$ with skew shapes $\gamma_{1}, \ldots, \gamma_{s}$, the height sign $\mathrm{ht}(T)$ is defined as $\mathrm{ht}(T)=\prod_{i=1}^{s}(-1)^{\mathrm{ht}\left(\gamma_{i}\right)}$.

For the border strip tableau in the above example, the heights are 5 (green), 1 (yellow), 2 (red), and 2 (blue), resulting in an overall height sign of +1 . Having established these preliminaries, we can state the Murnaghan-Nakayama rule. For proofs, we refer to textbooks [22, 25].

Theorem 10 (Murnaghan-Nakayama rule). For partitions $\lambda$ and $\rho$ and any ordering $\kappa$ of $\rho$, we have

$$
\chi_{\lambda}(\rho)=\sum_{T \in \mathcal{B}(\lambda, \kappa)} \operatorname{ht}(T) .
$$

Remark 11. When invoking this rule, it makes sense to choose a useful ordering $\kappa$ of $\rho$. For example, to show $\chi_{(5,4,3,2,1)}\left(1^{3}, 2^{6}\right)=0$, we can

- sum over a rather large number of border strip tableaux to observe that their signs cancel, or alternatively

- reorder $\rho=\left(1^{3}, 2^{6}\right)$ to $\kappa=\left(2^{6}, 1^{3}\right)$ and realize directly that no border strip on 2 boxes can be peeled from the border of the staircase $(5,4,3,2,1)$.

More generally, this observation shows that $\chi_{\mu}(\rho)=0$ whenever $\mu$ is a staircase and $\rho$ is a partition that contains at least one even part.

The above remark will be crucial for the staircase-based reduction in Section 5. For the tetromino-based reduction in Section 6, we extend Theorem 10 to character evaluations on products of partition sets.

Definition 12. Let $F_{1}, \ldots, F_{t}$ be sets of partitions such that each set $F_{i}$ collects partitions of the same integer $d_{i}$. The partition product $F_{1} \times \ldots \times F_{t}$ is the multi-set consisting of the $\prod_{i}\left|F_{i}\right|$ partitions of $d_{1}+\ldots+d_{t}$ obtained by choosing one partition from each set $F_{i}$ and concatenating those $t$ partitions.

We linearly extend class functions $f$ to multi-sets $S$ of partitions by declaring $f(S)=\sum_{\rho \in S} f(\rho)$ and show how to calculate $\chi_{\lambda}\left(F_{1} \times \ldots \times F_{t}\right)$ combinatorially by an extension of the Murnaghan-Nakayama rule. To this end, we define admissible skew shapes for each $F_{i}$, each with a particular coefficient. In analogy with the original rule, admissible skew shapes play the role of border strips, and the coefficients of admissible skew shapes play the role of heights of border strips.

Definition 13. Given a set of partitions $F$, let $\Gamma_{F}$ be the set of all skew shapes $\gamma$ that admit a border strip tableau $T \in \mathcal{B}(\gamma, \rho)$ for some $\rho \in F$. For $\gamma \in \Gamma_{F}$, we define the coefficient

$$
\alpha_{F}(\gamma)=\sum_{\rho \in F} \sum_{T \in \mathcal{B}(\gamma, \rho)} \operatorname{ht}(T)
$$

In preparation of Section 6, we exemplify this definition with the cycle formats of active edge gadgets. For $F=\left\{\left(2^{2}\right),(4)\right\}$, we observe that the set $\Gamma_{F}$ consists of all skew shapes that can be covered with two disjoint dominos. Note that there are shapes $\gamma \in \Gamma_{F}$ with $\alpha_{F}(\gamma)=0$ : For example, the vertical 4-box line admits a border strip tableau for $\left(2^{2}\right)$ and one for (4), shown below. These tableaux have opposite height signs and thus cancel.

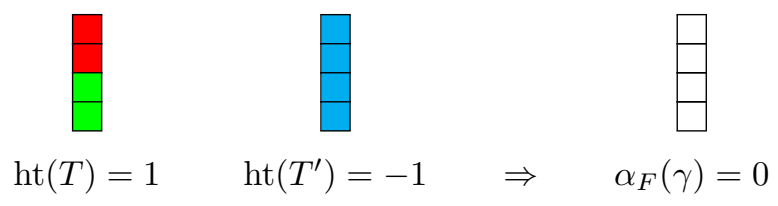


This outcome is expected: In Section 6, we show that 4-box shapes $\gamma$ with $\alpha_{F}(\gamma) \neq 0$ serve as a resource for establishing hardness of immanants. If we can peel many such shapes from a partition $\lambda$, then we can reduce a large permanent to the $\lambda$-immanant. Thus, if the vertical 4-box line $\gamma$ satisfied $\alpha_{F}(\gamma) \neq 0$, our reductions would allow us to establish hardness of the determinant.

With all relevant notions introduced, we can now turn to our generalization of the Murnaghan-Nakayama rule. The proof is almost syntactic; it essentially requires us to group border strips into skew shapes and collect terms accordingly. Nevertheless, a little care is required to ensure that the grouped border strips are indeed proper skew shapes.

Lemma 14. Let $\lambda$ be a partition. Given a partition product $F_{1} \times \ldots \times F_{t}$, abbreviate $\Gamma_{i}=\Gamma_{F_{i}}$ and $\alpha_{i}=\alpha_{F_{i}}$ for $i \in[t]$. Writing $\tilde{\Gamma}=\left(\Gamma_{1}, \ldots, \Gamma_{t}\right)$, we have

$$
\chi_{\lambda}\left(F_{1} \times \ldots \times F_{t}\right)=\sum_{\substack{S \in \mathcal{S}(\lambda, \tilde{\Gamma}) \\ \text { with shapes } \gamma_{1} \ldots \gamma_{t}}} \prod_{i=1}^{t} \alpha_{i}\left(\gamma_{i}\right) .
$$

Proof. Write $k_{i}:=\left|F_{i}\right|$ and enumerate $F_{i}=\left\{\rho_{i, 1}, \ldots, \rho_{i, k_{i}}\right\}$ for $i \in[t]$. We write $A=\left[k_{1}\right] \times \ldots \times\left[k_{t}\right]$ for the set of multi-indices into $F_{1} \times \ldots \times F_{t}$. For any multi-index $a \in A$, we define the ordered partition

$$
\rho_{a}:=\left(\rho_{1, a(1)}, \ldots, \rho_{t, a(t)}\right)
$$

by concatenating the partitions $\rho_{i, a(i)}$ for $i \in[t]$. Here, the order within $\rho_{i, a(i)}$ is not relevant, but the partitions need to be concatenated in the specified order. We then have

$$
\chi_{\lambda}\left(F_{1} \times \ldots \times F_{t}\right)=\sum_{a \in A} \sum_{T \in \mathcal{B}\left(\lambda, \rho_{a}\right)} \operatorname{ht}(T) .
$$

Given $a \in A$ and a border strip tableau $T \in \mathcal{B}\left(\lambda, \rho_{a}\right)$, we define the skew shape tableau $S(T)$ by grouping, for each $i \in[t]$, the different border strips corresponding to block $\rho_{i, a(i)}$ into a skew shape. Since these border strips are peeled consecutively in $T$, the tableau $S(T)$ is indeed a skew shape tableau, and it is contained in $\mathcal{S}(\lambda, \tilde{\Gamma})$ by definition of the sets $\Gamma_{1}, \ldots, \Gamma_{t}$. We then partition the set of tableaux $T \in \mathcal{B}\left(\lambda, \rho_{a}\right)$ according to $S(T)$ and obtain

$$
\sum_{a \in A} \sum_{T \in \mathcal{B}\left(\lambda, \rho_{a}\right)} \operatorname{ht}(T)=\sum_{a \in A} \sum_{S \in \mathcal{S}(\lambda, \tilde{\Gamma})} \sum_{\substack{T \in \mathcal{B}\left(\lambda, \rho_{a}\right) \\ \text { with } S(T)=S}} \operatorname{ht}(T) .
$$

Let us change the order of summation and sum over $S \in \mathcal{S}(\lambda, \tilde{\Gamma})$ first. For a fixed skew shape tableau $S$ with shapes $\gamma_{1}, \ldots, \gamma_{t}$, each border strip tableau $T \in \bigcup_{a \in A} \mathcal{B}\left(\lambda, \rho_{a}\right)$ with $S(T)=S$ is obtained by choosing a border strip tableau independently for each of the shapes $\gamma_{1}, \ldots, \gamma_{t}$. We obtain

$$
\sum_{a \in A} \sum_{\substack{T \in \mathcal{B}\left(\lambda, \rho_{a}\right) \\ \text { with } S(T)=S}} \operatorname{ht}(T)=\prod_{i=1}^{t} \sum_{j=1}^{k_{i}} \sum_{T \in \mathcal{B}\left(\gamma_{i}, \rho_{i, j}\right)} \operatorname{ht}(T)=\prod_{i=1}^{t} \alpha_{i}\left(\gamma_{i}\right) .
$$

By summing both sides over all $S \in \mathcal{S}(\lambda, \tilde{\Gamma})$ and combining the result with (5) and (4), the lemma follows.

\section{Staircases versus non-vanishing tetrominos}

We associate various quantities with partitions $\lambda \vdash n$ to measure to what extent the $\lambda$-immanant lends itself to a reduction from counting matchings. Then we investigate their interplay with the number $b(\lambda)$ of boxes to the right of the first column of $\lambda$.

The diagrams $\square$ and $\boxminus$ of $\left(2^{1}\right)$ and $\left(1^{2}\right)$ will be denoted as dominos. The domino number $d(\lambda)$ is the maximum number of dominos that can be peeled successively from $\lambda$. As we show below, the result of peeling 
these dominos from $\lambda$ is a partition of the form $\mu=(k, \ldots, 1)$ for some $k \in \mathbb{N}$. Such partitions and their associated shapes are called staircases, and we define $z(\lambda)=k+\ldots+1$ as the staircase size and $w(\lambda)=k$ as the staircase width of $\lambda$. Note that $2 d(\lambda)+z(\lambda)=n$ and that $z(\lambda)$ can be zero; this happens when $\lambda$ can be covered fully by dominos.

Fact 15. The shape $\mu$ obtained by peeling $d(\lambda)$ dominos from $\lambda$ is the unique staircase on $z(\lambda)$ boxes.

Proof. By maximality of $d(\lambda)$, no domino can be peeled from $\mu$, so all gaps between consecutive rows in $\mu$ are 1 . This requires $\mu$ to be a staircase. It has $n-2 d(\lambda)=z(\lambda)$ boxes, and the number of boxes uniquely determines a staircase.

We define $s(\lambda)$ to be the maximum number of non-vanishing tetrominos (depicted on page 5 and Table 2) that can be peeled successively from $\lambda$. As we establish in Sections 5 and 6 , any partition $\lambda$ with large $w(\lambda)$ or $s(\lambda)$ induces an immanant to which the hard problem of counting $k$-matchings can be reduced. In this subsection, we show that at least one of these numbers is large if $b(\lambda)$ is large. Towards this, we first observe that partitions $\lambda$ with $s(\lambda)=0$ are strongly restricted.

Lemma 16. For any partition $\lambda$ with $s(\lambda)=0$, at least one of the following holds:

1. The shape $\lambda$ is a staircase up to some number of additional boxes in the first column.

2. There is an index $i^{*}$ such that, after peeling a horizontal domino from each row $i \leq i^{*}$, the remaining shape is the staircase of $\lambda$.

Proof. There is at most one row $i$ with gap $\delta_{i}>1$ : If $\delta_{i}, \delta_{i^{\prime}} \geq 2$ for $i \neq i^{\prime}$, then we could peel $\square$ from these two rows. As these dominos share no rows or columns, they constitute a non-vanishing tetromino, contradicting $s(\lambda)=0$. Similarly, we see that the set of rows $i$ with $\delta_{i}=0$, if non-empty, forms a contiguous interval $[j, k]$ : If the set contained two disjoint intervals, we could peel two $\boxminus$ from their ends.

In the following, we distinguish whether $\lambda$ contains a row with gap 0 or not. If such a row exists, we show that the first case of the lemma statement applies. Otherwise, we establish the second case.

1. If there is a row $i$ with gap $\delta_{i}=0$, let $[j, k] \ni i$ be the single interval of rows with gap 0 . We rule out the existence of a row $i^{\prime}$ with $\delta_{i^{\prime}}>1$, as it would lead to the following contradictions:

- If $i^{\prime}<j$ or $i^{\prime}>k+1$, then we could peel $\square$ from row $i^{\prime}$ and $\boxminus$ from rows $k-1$ and $k$. This contradicts $s(\lambda)=0$.

- If $i^{\prime}=k+1$, then we could peel $\boxplus$ from rows $k$ and $k+1$.

It follows that $\delta_{i^{\prime}}=1$ for all $i^{\prime} \notin[j, k]$. Next, we show that $\lambda_{i}=1$ for all $i \in[j, k]$ : If $\lambda_{j}=\lambda_{k}>1$, then $\boxminus$ could be peeled from rows $k$ to $k+2$ for a contradiction. (Indeed, we would have $\delta_{k+1}=1$, since $\delta_{i^{\prime}}=1$ for all rows $i^{\prime} \notin[j, k]$, and $\lambda_{k}>1$ would that row $k+2$ exists.) Hence, the first case of the lemma applies.

2. Otherwise, we have $\delta_{i}>0$ for all rows $i$. As discussed in the first paragraph of the proof, there is at most one row $i^{*}$ with $\delta_{i^{*}}>1$.

- If $\delta_{i^{*}} \geq 4$, we could peel $\square$ from row $i^{*}$, contradicting $s(\lambda)=0$.

- If $\delta_{i^{*}}=2$ and row $i^{*}+1$ exists, we could peel $\square$ from rows $i^{*}$ and $i^{*}+1$.

It follows that $\delta_{i^{*}}=3$, or that $i^{*}$ is the last row and $\delta_{i^{*}}=2$. As $i^{*}$ is the only row with gap $\delta_{i^{*}} \neq 1$, the second case of the lemma applies.

Since at least one of the two above cases applies, the lemma is proven.

It follows that partitions $\lambda$ with $s(\lambda)=0$ contain only few boxes outside their first column and staircase.

Corollary 17. Let $\lambda$ be a partition with $s(\lambda)=0$ and staircase $\mu$ of width $w \in \mathbb{N}$. Then $b(\lambda / \mu) \leq 2 w+1$. 
Proof. If the second case of Lemma 16 applies to $\lambda$, then $b(\lambda / \mu) \leq 2 w+1$. If the first case applies, let $\mu^{\prime}$ denote the largest staircase contained in $\lambda$. (This need not be the staircase of $\lambda$.) Then $\lambda$ is $\mu^{\prime}$ with some number $a \in \mathbb{N}$ of additional boxes in the first column. When peeling dominos from $\lambda$, the first dominos must be contained in the first column.

- If $a$ is even, then $\mu^{\prime}$ is the staircase of $\lambda$, so $b(\lambda / \mu)=0$ : Peeling $\boxminus$ from the first column results in $\mu^{\prime}$.

- If $a$ is odd, then the lowest box of $\mu^{\prime}$ is contained in the last $\boxminus$ that can be peeled from the first column. We can then peel a horizontal domino from each of the remaining $w+1$ rows. The remainder is the staircase of $\lambda$. It follows that $b(\lambda / \mu) \leq 2 w+1$.

In both cases, we obtain $b(\lambda / \mu) \leq 2 w+1$, thus proving the corollary.

We are ready to prove the main lemma of this section.

Lemma 18. For any partition $\lambda$, at least one of $s(\lambda) \geq b(\lambda) / 8$ or $w(\lambda) \geq \sqrt{b}-1$ holds.

Proof. Let $\mu$ be the staircase of $\lambda$ and $w=w(\lambda)$. The number of boxes to the right of the first column in $\lambda / \mu$ is $b(\lambda / \mu)=b(\lambda)-b(\mu)=b(\lambda)-\left(\begin{array}{c}w \\ 2\end{array}\right)$. By applying the contraposition of Corollary 17 repeatedly, it follows that

We obtain

$$
s(\lambda) \geq \frac{b(\lambda / \mu)-(2 w+1)}{4}=\frac{b(\lambda)-\left(\begin{array}{c}
w \\
2
\end{array}\right)-(2 w+1)}{4}=\frac{b(\lambda)}{4}-\frac{w^{2}+3 w+2}{8} .
$$

$$
s(\lambda)+\frac{w^{2}+3 w+2}{8} \geq \frac{b(\lambda)}{4},
$$

and hence, at least one of the two terms is larger than $b(\lambda) / 8$. This implies the lemma.

\section{$5 \quad$ Exploiting a staircase}

We show how to count $k$-matchings in $n$-vertex graphs $H$ with access to the $\lambda$-immanant for a partition $\lambda$ with staircase size $z(\lambda) \in \Omega(k)$. If $k \ll n$, we also require a large domino number $d(\lambda)$. Our reduction relies on the intermediate problem of counting cycle covers with a particular "onion" format, outlined in Section 5.1, which we then reduce to the $\lambda$-immanant using a graph construction that has a favorable interplay with staircase characters, as established in Section 5.2. We collect the steps in Section 5.3.

\subsection{Onion partitions}

We first describe a particular "onion partition" derived from $\lambda$, depicted in Figure 1. To define this partition, recall that $w(\lambda)$ and $z(\lambda)$ denote the staircase width and size of $\lambda$, respectively.

Definition 19. Let $\lambda \vdash n^{\prime}$ be a partition with staircase $\mu$. For $\ell \leq w(\lambda) / 2$, let $\theta$ be the partition obtained by peeling $\ell$ maximal-length border strips from $\mu$ and recording their lengths. We write $\|\theta\|$ for the number of boxes in $\theta$. Then the $\ell$-layer onion $\rho$ of $\lambda$ is the partition $\left(2^{d(\lambda)}, \theta, 1^{z(\lambda)-\|\theta\|}\right)$. For $k \in \mathbb{N}$, we say that $\rho$ accommodates $k$ edges if $\|\theta\| \geq 2 k+\ell$.

In words, the $\ell$-layer onion $\rho$ of $\lambda$ is obtained as follows: First peel all $d(\lambda)$ dominos from $\lambda$ to expose the staircase $\mu$. Then peel $\ell$ maximal-length border strips from $\mu$. Finally, peel the remaining $z(\lambda)-\|\theta\|$ boxes of $\mu$ as singletons. If $\rho$ accommodates $k$ edges, then the border strips contain $2 k$ boxes and one extra box for each peeled border strip; such partitions allow us to reduce counting $k$-matchings to counting $\rho$-cycle covers.

Next, we describe two related ways of constructing a digraph $G$ whose cycle covers of onion format correspond to $k$-matchings in $H$. Given an edge-weighted digraph $G$, let

$$
\# \mathrm{CC}(G, \rho)=\sum_{\substack{\rho \text {-cycle cover } \\ C \text { in } G}} \prod_{e \in C} w(e) .
$$




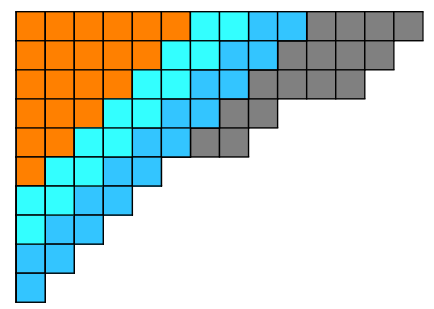

Figure 1: A partition $\lambda$ with $d(\lambda)=8$ dominos, staircase width $w(\lambda)=10$ and size $z(\lambda)=55$. The 2-layer onion $\rho=\left(2^{8}, 19,15,1^{21}\right)$ of $\lambda$ is shown as a border strip tableau; parts corresponding to dominos and singletons are not distinguished in this figure. All border strip tableaux for $\rho$ in $\lambda$ have the depicted form. This onion accommodates 16 edges; the two layers contain 34 boxes, and one box per layer is required for "closing" the layer.
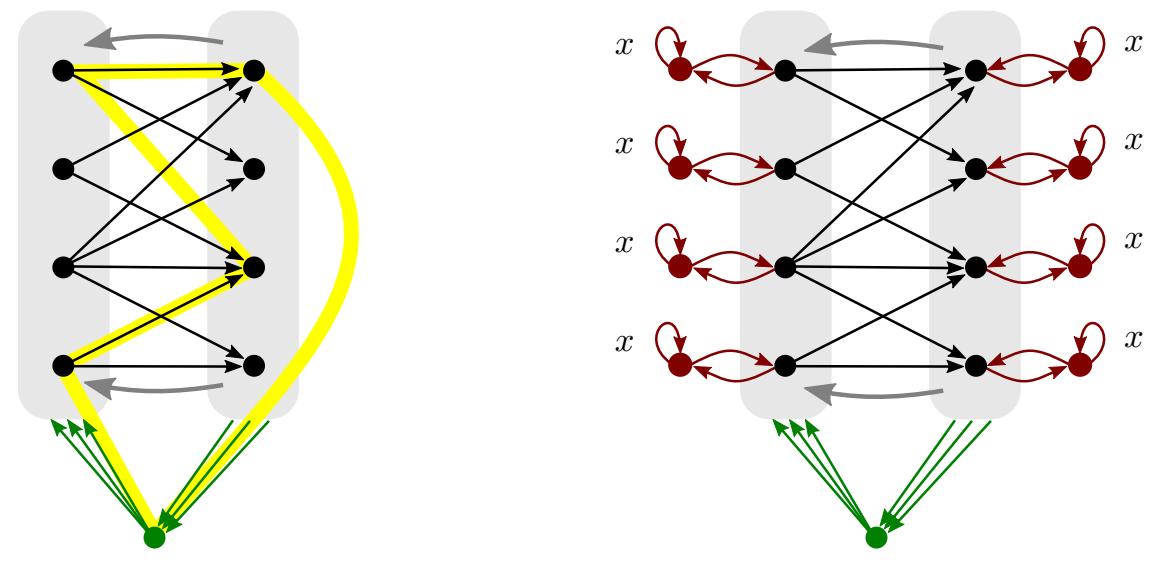

Figure 2: The graphs from Lemma 20. To reduce clutter, edges from $R$ to $L$ are only hinted, and only one transit vertex is shown. In the left graph, a cycle corresponding to a 2-matching is displayed in yellow color.

Furthermore, we write $\left[x^{s}\right] p$ for the coefficient of $x^{s}$ in a polynomial $p \in \mathbb{Q}[x]$. We also say that a vertex-set $T$ in a graph $G$ is an odd-cycle transversal if $G-T$ is bipartite up to self-loops. (Note that not requiring self-loops to be removed by $T$ may not be the standard way of defining odd-cycle transversals.)

Lemma 20. Let $H$ be a bipartite $n$-vertex graph and let $\lambda$ be an integer partition.

1. If there are onions of $\lambda$ that accommodate $n / 2$ edges, let $\rho$ be such an onion with the minimal number of layers. In polynomial time, we can construct a digraph $G$ with $\# \mathrm{CC}(G, \rho)=(n / 2) ! \cdot \# \operatorname{PerfMatch}(H)$.

2. For any $k \in \mathbb{N}$ with $d(\lambda) \geq n-2 k$ : If there are onions of $\lambda$ that accommodate $k$ edges and contain $\geq 2 k$ copies of 1 , let $\rho$ be such an onion with the minimal number of layers. In polynomial time, we can construct a digraph $G$ (whose edge-weights may be constant multiples of an indeterminate $x$ ) such that $\left[x^{2 k}\right] \# \mathrm{CC}(G, \rho)=k ! \cdot \# \operatorname{Match}(H, k)$.

In both cases, let $\rho=\left(2^{d(\lambda)}, \theta, 1^{z(\lambda)-\|\theta\|}\right)$ be the relevant $\ell$-layer onion, for $\ell \in \mathbb{N}$. Then the following holds:

- $G$ admits an odd-cycle transversal $T \subseteq V(G)$ of cardinality $\ell$.

- Every cycle cover in $G$ (in the second case, every cycle cover whose weight is a constant multiple of $\left.x^{2 k}\right)$ contains exactly $z(\lambda)-\|\theta\|$ self-loops and at least $d(\lambda)$ digons.

Proof. Let $V(H)=L \cup R$ and write $\rho=\left(2^{d(\lambda)}, \theta, 1^{z(\lambda)-\|\theta\|}\right)$ for the relevant $\ell$-layer onion of $\lambda$. By adding isolated edges to $H$, we assume that $n / 2$ (in the first case) or $k$ (in the second case) is the maximum number of edges accommodated by $\lambda$. For the first part of the lemma, the graph $G$ is defined as follows: 
1. Direct all edges in $H$ from $L$ to $R$ and add all edges $R \times L$.

2. Add a set of transit vertices $T=\left\{t_{1}, \ldots t_{\ell}\right\}$ and all edges in $R \times T$ and $T \times L$.

3. Add $d(\lambda)$ disjoint padding digons and $z(\lambda)-\|\theta\|$ padding vertices with self-loops.

Any $\rho$-cycle cover of $G$ uses all padding digons and loops. Any remaining odd-length cycle must use a vertex of $T$, as it would otherwise be contained in a bipartite graph. This implies that $T$ is an odd-cycle transversal. It follows that the $\ell$ (odd-length) parts in $\theta$ can only be accommodated by $\ell$ disjoint cycles in $G$ that each include exactly one transit vertex. Hence, any $\rho$-cycle cover $C$ in $G$ induces a perfect matching in $H$ when restricted to edges from $L$ to $R$ : Deleting transit vertices results in a cover of $V(H)$ with paths of odd length $\geq 1$ that start in $L$, and deleting the edges from $R$ to $L$ then leaves us with a matching.

Conversely, every perfect matching in $H$ induces exactly $(n / 2)$ ! cycle covers of format $\rho$ in $G$ : For $\theta=\left(2 r_{1}+1,2 r_{2}+1, \ldots\right)$, there are $\left(\begin{array}{c}n / 2 \\ r_{1}, \ldots, r_{\ell}\end{array}\right)$ ways of partitioning the $n / 2$ matching edges into the cycles corresponding to transit vertices $t_{1}, \ldots t_{\ell}$, and there are $r_{i}$ ! ways of choosing an ordering of the edges and the $i$-th transit vertex within the $i$-th cycle. ${ }^{3}$ This concludes the first part of the lemma.

For the second part, we construct $G$ by performing steps 1 and 2 from above, followed by these steps:

3. For each vertex $v \in V(H)$, add a switch vertex $s_{v}$ with a self-loop of weight $x$, and a switch digon between $v$ and $s_{v}$.

4. Add $d(\lambda)-(n-2 k)$ padding digons and $z(\lambda)-\|\theta\|-2 k$ padding vertices with self-loops.

Again, any cycle cover $C$ of $G$ uses all padding elements. The weight of $C$ is $x^{2 k}$ iff it includes exactly $2 k$ self-loops at switch vertices; it then includes $n-2 k$ switch digons touching the remaining switch vertices, so it contains at least $d(\lambda)$ digons and $z(\lambda)-\|\theta\|$ self-loops. As before, if $C$ has format $\rho$, then the $\ell$ remaining odd-length cycles induce a $k$-matching in $H$ when restricted to edges from $L$ to $R$, and any $k$-matching in $H$ can be extended to $k$ ! such cycle covers.

The odd-cycle transversal of $G$ and special properties of staircase characters ensure that we can determine \#CC $(G, \rho)$ for an onion $\rho$ of $\lambda$ by reduction to $\operatorname{imm}_{\lambda}(G)$. We prove this in the next subsection.

\subsection{Staircase characters}

Given a partition $\lambda$ with staircase $\mu$, consider any partition $\rho=\left(2^{d(\lambda)}, \rho^{\prime}\right)$ obtained by peeling the maximum number of dominos from $\lambda$, followed by some other partition $\rho^{\prime}$ of the staircase $\mu$ thusly exposed. We show how to relate $\chi_{\lambda}(\rho)$ to $\chi_{\mu}\left(\rho^{\prime}\right)$ by means of domino tilings of $\lambda / \mu$, which are border strip tableaux that only contain the dominos $\boxminus$ and $\square$.

Definition 21. A domino tiling of a skew shape $\lambda / \mu$ is a border strip tableau $T$ of $\lambda / \mu$ with format $\left(2^{\|\lambda / \mu\| / 2}\right)$. The parity of a domino tiling $T$ is the parity of the number of $\boxminus$ in $T$.

Note that the parity of a domino tiling $T$ is essentially its height $\operatorname{sign} \operatorname{ht}(T)$ : The parity of $T$ is even/odd iff the height sign of $T$ is positive/negative. Curiously, these numbers do not depend on $T$.

Lemma 22. All domino tilings of a fixed shape $\lambda / \mu$ have the same parity.

Proof. Paint the rows of $\lambda / \mu$ black and white in an alternating way. In any domino tiling $T$, every vertical (horizontal) domino contains an odd (even) number of white boxes. Hence, the number of vertical dominos in $T$ agrees in parity with the number of white boxes in $\lambda / \mu$, which does not depend on $T$.

This elementary yet crucial observation gives the desired connection between $\chi_{\lambda}(\rho)$ and $\chi_{\mu}\left(\rho^{\prime}\right)$.

\footnotetext{
${ }^{3}$ Each such ordering corresponds to a pair $(j, \sigma)$ for $j \in\left[r_{i}\right]$ and a cyclic permutation $\sigma$ of $\left[r_{j}\right]$. The index $j$ indicates that the transit vertex $t_{i}$ is visited immediately after the $j$-th edge, and $\sigma$ describes the order of the edges along the cycle. There are $r_{i} \cdot\left(r_{i}-1\right) !=r_{i}$ ! such pairs $(j, \sigma)$.
} 
Lemma 23. Let $\lambda$ be a partition with staircase $\mu$, and let $\rho=\left(2^{d(\lambda)}, \rho^{\prime}\right)$ for a partition $\rho^{\prime}$. Then we have $\chi_{\lambda}(\rho) \neq 0$ iff $\chi_{\mu}\left(\rho^{\prime}\right) \neq 0$.

Proof. As any way of peeling $d(\lambda)$ dominos from $\lambda$ results in $\mu$, the Murnaghan-Nakayama rule (Theorem 10) shows that $\chi_{\lambda}(\rho)=c_{\lambda / \mu} \cdot \chi_{\mu}\left(\rho^{\prime}\right)$ with

$$
c_{\lambda / \mu}=\sum_{\substack{\text { domino tiling } \\ T \text { of } \lambda / \mu}} \operatorname{ht}(T),
$$

By Lemma 22, each term in (6) has the same sign. Since $\lambda / \mu$ is obtained by peeling $d(\lambda)$ dominos from $\lambda$, there is at least one domino tiling, and hence there is at least one term in the sum. It follows that $c_{\lambda / \mu}>0$, thus proving the lemma.

The last missing piece is to recall from Remark 11 that the staircase character $\chi_{\mu}\left(\rho^{\prime}\right)$ vanishes whenever $\rho^{\prime}$ contains an even part. This allows us to analyze cycle covers in the particular graphs $G$ constructed in the last subsection: Any such cycle cover (with the right number of self-loops and digons) is counted by $\operatorname{imm}_{\lambda}(G)$ iff its format is an onion.

Lemma 24. Let $\lambda \vdash n^{\prime}$ be a partition and let $\rho=\left(2^{d(\lambda)}, \theta, 1^{z(\lambda)-\|\theta\|}\right)$ be the $\ell$-layer onion of $\lambda$. Let $G$ be an $n^{\prime}$-vertex digraph with an odd-cycle transversal of size $\ell$. For any cycle cover $C$ in $G$ that contains at least $d(\lambda)$ digons and exactly $z(\lambda)-\|\theta\|$ self-loops: If $\chi_{\lambda}(C) \neq 0$, then the format of $C$ is $\rho$.

Proof. Let $C$ be a cycle cover in $G$ with format $\beta=\left(2^{d(\lambda)}, \rho^{\prime}\right)$ and $\chi_{\lambda}(\beta) \neq 0$. By Lemma 23 , we have $\chi_{\mu}\left(\rho^{\prime}\right) \neq 0$, where $\mu$ is the staircase of $\lambda$. Since $G$ has an odd-cycle transversal of size $\ell$, there are at most $\ell$ non-singleton odd parts in $\rho^{\prime}$, as every non-singleton odd cycle uses exactly one transversal vertex. Since $\rho^{\prime}$ contains $z(\lambda)-\|\theta\|$ singletons, the non-singleton odd parts contain $\|\theta\|$ boxes in total. This can only be achieved by peeling $\ell$ maximal-length border strips from $\mu$, so the cycle format of the non-singleton parts is $\theta$. Thus, we have $\rho^{\prime}=\left(\theta, 1^{z(\lambda)-\|\theta\|}\right)$.

Corollary 25. Let $\lambda \vdash n^{\prime}$ and let $\rho=\left(2^{d(\lambda)}, \theta, 1^{z(\lambda)-\|\theta\|}\right)$ be the $\ell$-layer onion of $\lambda$. Let $G$ be an $n^{\prime}$-vertex graph with an odd-cycle transversal of size $\ell$. For $t \in \mathbb{N}$, if every cycle cover whose weight is a constant multiple of $x^{t}$ contains at least $d(\lambda)$ digons and $z(\lambda)-\|\theta\|$ self-loops, then

$$
\left[x^{t}\right] \operatorname{imm}_{\lambda}(G)=\chi_{\lambda}(\rho) \cdot\left[x^{t}\right] \# \operatorname{CC}(G, \rho)
$$

with $\chi_{\lambda}(\rho) \neq 0$.

Proof. The equation follows directly from Lemma 24 and the requirement on $G$. To show $\chi_{\lambda}(\rho) \neq 0$, it suffices by Lemma 23 to show $\chi_{\mu}\left(\rho^{\prime}\right) \neq 0$ for $\rho^{\prime}=\left(\theta, 1^{z(\lambda)-\|\theta\|}\right)$. There is exactly one way of peeling $\theta$ from $\rho^{\prime}$, and each of the $z(\lambda)-\|\theta\|$ singletons peeled from the resulting staircase incurs positive height sign. It follows that all border strip tableaux of format $\rho^{\prime}$ in $\mu$ contribute to $\chi_{\mu}\left(\rho^{\prime}\right)$ with the same sign.

Note that we can consider $G$ to be unweighted and invoke the corollary with $t=0$. This yields the unweighted setting (in $x$ ) from the first part of Lemma 20.

\subsection{Reductions}

We combine the results from the previous sections into reductions from counting matchings to evaluating immanants for partitions with large staircases.

Lemma 26. The following can be achieved in polynomial time and with polynomial-sized arithmetic circuits:

1. Given a bipartite $n$-vertex graph $H$ and a partition $\lambda$ with $w(\lambda) \geq 2 \sqrt{n}$, compute a digraph $G$ and $a$ number $c \in \mathbb{Q}$ such that \#PerfMatch $(H)=c \cdot \operatorname{imm}_{\lambda}(G)$. 
2. Given a bipartite $n$-vertex graph $H$ and $k \in \mathbb{N}$, as well as a partition $\lambda$ with $w(\lambda) \geq 4 \sqrt{k}$ and $d(\lambda) \geq n-2 k$, compute a digraph $G$ and a number $c \in \mathbb{Q}$ such that \#Match $(H, k)=c \cdot\left[x^{k}\right] \operatorname{imm}_{\lambda}(G)$.

Proof. For the first part, note that $w(\lambda) \geq 2 \sqrt{n}$ implies that $\lambda$ admits a $t$-layer onion $\rho=\left(2^{d(\lambda)}, \theta, 1^{z(\lambda)-\|\theta\|}\right)$ for $t=\lfloor\sqrt{n}\rfloor$. For large enough $n$, the number of edges accommodated by this onion is at least

$$
\frac{\left(\begin{array}{c}
2 t+1 \\
2
\end{array}\right)-t}{2}=t^{2} \geq n-2 \sqrt{n}+1 \geq n / 2 .
$$

Hence, Lemma 20 allows us to construct a digraph $G$ with $\# \mathrm{CC}(G, \rho)=(n / 2) ! \cdot \# \operatorname{PerfMatch}(H)$ such that $G$ admits an odd-cycle transversal of cardinality $t$, and every cycle cover in $G$ contains $z(\lambda)-\|\theta\|$ self-loops and at least $d(\lambda)$ digons. By Corollary 25, we then have $\operatorname{imm}_{\lambda}(G)=\chi_{\lambda}(\rho) \cdot \# \operatorname{CC}(G, \rho)$ with $\chi_{\lambda}(\rho) \neq 0$. It follows from the last two equations that

$$
\# \operatorname{PerfMatch}(H)=\frac{\operatorname{imm}_{\lambda}(G)}{(n / 2) ! \cdot \chi_{\lambda}(\rho)} .
$$

Note that $\chi_{\lambda}(\rho)$ can be computed in polynomial time and with polynomial-sized arithmetic circuits, see Proposition 7.4 in [8].

For the second part, note that $w(\lambda) \geq 4 \sqrt{k}$ implies that $\lambda$ admits an onion $\rho=\left(2^{d(\lambda)}, \theta, 1^{z(\lambda)-\|\theta\|}\right)$ that accommodates $\geq k$ edges. Additionally, $w(\lambda) \geq 4 \sqrt{k}$ also implies that $z(\lambda)-\|\theta\| \geq 2 k$. Since $d(\lambda) \geq n-2 k$ holds by assumption, all conditions for the second part of Lemma 20 are thus fulfilled, and we can construct a graph $G$ such that $\left[x^{2 k}\right] \# \mathrm{CC}(G, \rho)=k ! \cdot \# \operatorname{Match}(H, k)$ and any cycle cover of weight $x^{2 k}$ in $x$ contains exactly $z(\lambda)-\|\theta\|$ self-loops and at least $d(\lambda)$ digons. Corollary 25 then implies that $\left[x^{2 k}\right] \operatorname{imm}_{\lambda}(G)=\chi_{\lambda}(\rho) \cdot\left[x^{2 k}\right] \# \mathrm{CC}(G, \rho)$ with $\chi_{\lambda}(\rho) \neq 0$. We conclude that

$$
\# \operatorname{Match}(H, k)=\frac{\left[x^{2 k}\right] \operatorname{imm}_{\lambda}(G)}{k ! \cdot \chi_{\lambda}(\rho)} .
$$

This proves the lemma.

\section{Exploiting non-vanishing tetrominos}

We show how to count $k$-matchings with access to $\lambda$-immanants for partitions $\lambda$ with large non-vanishing tetromino number $s(\lambda)$ : In Section 6.1, we use edge gadgets to construct particular immanants $\operatorname{imm}_{\lambda}(G)$ that count matchings up to a multiplicative constant factor. We then show, in Section 6.2, that the factor arising in the above construction is non-zero. To this end, we prove that the character $\chi_{\lambda}$ does not vanish on a particular partition product. Finally, we combine these insights in Section 6.3 to obtain a reduction from counting matchings.

\subsection{Main construction}

Consider the edge gadget $Q$ depicted on page 6 . Intuitively speaking, this graph fragment ensures that unwanted cycle covers in $G$ annihilate in $\operatorname{imm}_{\lambda}(G)$. In the remaining consistent cycle covers $C$, the edges within each edge gadget $Q$ cover either both endpoints (in an active state, as listed in Figure 3) or none of them (in a passive state). In particular, this allows us to interpret an active gadget $Q$ as a matching edge between its endpoints, since the active state prevents any edge outside of $Q$ from being incident with the endpoints of $Q$. A similar approach was taken by the author together with Bläser [3] to establish hardness of the so-called cover polynomial.

Lemma 27. Let $G$ be a directed graph containing copies $Q_{1}, \ldots, Q_{t}$ of the edge-gadget $Q$, with endpoints $e_{i}=\left\{u_{i}, v_{i}\right\}$ for $i \in[t]$, such that distinct edge-gadgets intersect only at endpoints. Let $\mathcal{C}^{*}(G)$ denote the set 


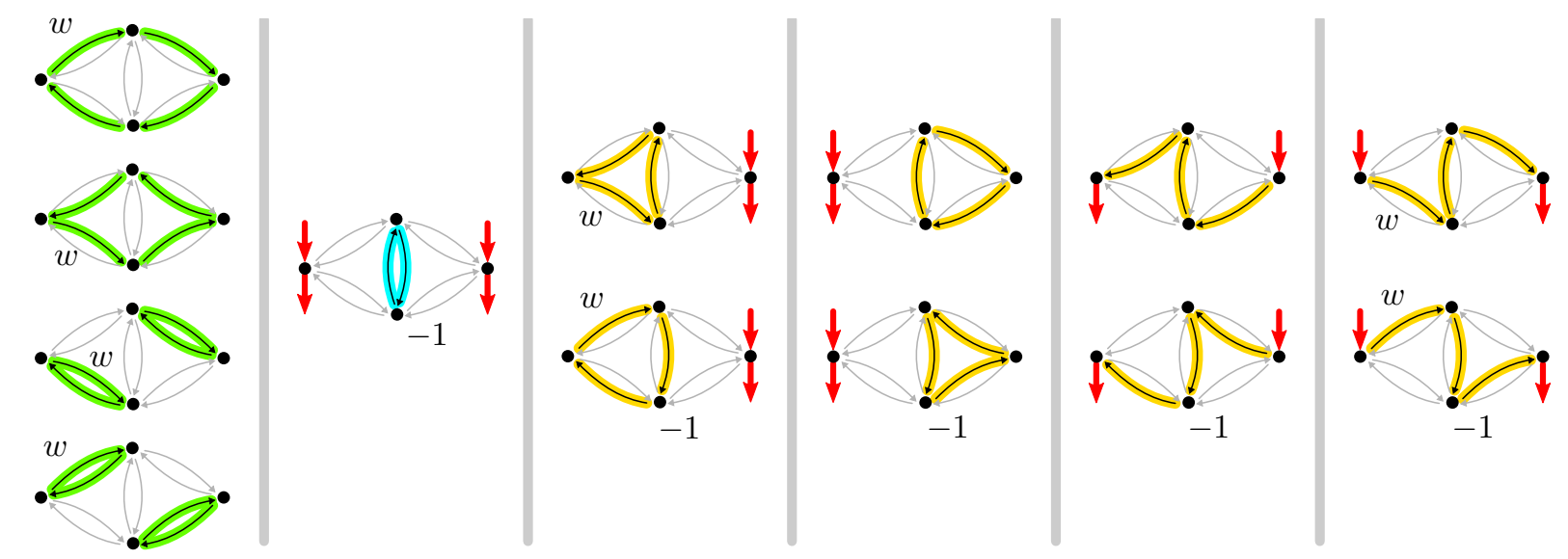

Figure 3: Each column lists the edge-sets $C_{i}$ that can arise for fixed combinations of endpoint in/out-degrees at gadget $Q_{i}$ in $E(G) \backslash E\left(Q_{i}\right)$. The first column lists active states, the second column shows the passive state. All other states come with annihilating partners and cancel out, as shown in Lemma 27.

of consistent cycle covers $C$ in $G$ : In such cycle covers, the restriction $C \cap E\left(Q_{i}\right)$ for $i \in[t]$ is an active or passive state, as depicted in Figure 3. Then we have

$$
\operatorname{imm}_{\lambda}^{*}(G):=\sum_{C \in \mathcal{C}^{*}(G)} \chi_{\lambda}(C) \prod_{e \in C} w(e)=\operatorname{imm}_{\lambda}(G) .
$$

Proof. For $i \in[t]$, we say that edges from $E(G) \backslash E\left(Q_{i}\right)$ are $i$-external. For any cycle cover $C \in \mathcal{C}(G)$, the total number of $i$-external incoming edges at the endpoints $u_{i}$ and $v_{i}$ of $Q_{i}$ equals the total number of $i$-external outgoing edges at $u_{i}$ and $v_{i}$ : Otherwise, the vertices of $Q_{i}$ cannot be covered by cycles. This gives six possible in/out-degree combinations at $u_{i}$ and $v_{i}$. For each of these combinations, the possible restrictions $C \cap E\left(Q_{i}\right)$ are listed in Figure 3 .

Using this list, we show that the set $\mathcal{C} \backslash \mathcal{C}^{*}$ of non-consistent cycle covers can be partitioned into pairs $\{C, \bar{C}\}$ whose constituents have equal cycle formats $\rho(C)=\rho(\bar{C})$ and opposite weights $w(C)=-w(\bar{C})$. Indeed, given $C \in \mathcal{C} \backslash \mathcal{C}^{*}$, let $i \in[t]$ be minimal such that $C_{i}=C \cap E\left(Q_{i}\right)$ is not consistent. Then $C_{i}$ corresponds to one of the edge-sets in the last four columns of Figure 3. Exchanging $C_{i}$ for the other edge-set in its column yields a cycle cover $\bar{C} \in \mathcal{C} \backslash \mathcal{C}^{*}$ of the same cycle format as $C$, but with opposite weight. We thus have $\chi_{\lambda}(C) \cdot w(C)=-\chi_{\lambda}(\bar{C}) \cdot w(\bar{C})$. As the map from $C$ to $\bar{C}$ induces a fixed-point free involution on $\mathcal{C} \backslash \mathcal{C}^{*}$, the pairs $\{C, \bar{C}\}$ indeed partition $\mathcal{C} \backslash \mathcal{C}^{*}$, and we conclude that

$$
\operatorname{imm}_{\lambda}(G)=\sum_{C \in \mathcal{C}^{*}} \chi_{\lambda}(C) \cdot w(C)+\underbrace{\sum_{C \in \mathcal{C} \backslash \mathcal{C}^{*}} \chi_{\lambda}(C) \cdot w(C)}_{=0},
$$

which proves the lemma.

Using this lemma, we show how to transform instances $(H, k)$ for \#Match into digraphs $G$ such that $\operatorname{imm}_{\lambda}(G)$ equals \#Match $(H, k)$ up to a multiplicative constant. Our constructions only rely on non-vanishing tetrominos and dominos; the staircase of $\lambda$ is "discarded" by introducing $z(\lambda)$ padding vertices with self-loops. The constant arising in this construction needs some attention. To define it, consider the partition products $\left\{\left(2^{2}\right),(4)\right\}^{\times s}$ induced by the cycle formats of active states; we pad the partitions in this product to partitions of $n^{\prime}$ with $d-2 s$ dominos and $z(\lambda)$ singletons. Formally, for fixed $\lambda \vdash n^{\prime}$ and $s \leq s(\lambda)$, define

$$
\theta_{s}:=\left\{\left(2^{2}\right),(4)\right\}^{\times s} \times\left\{\left(2^{d(\lambda)-2 s}, 1^{z(\lambda)}\right)\right\} .
$$

In the next subsection, we then establish the crucial fact that $\chi_{\lambda}\left(\theta_{s}\right) \neq 0$ for relevant choices of $s$. 
Lemma 28. Let $H$ be a graph with $n$ vertices and $m$ edges and let $\lambda \vdash n^{\prime}$ be a partition.

1. If $\lambda$ has skew tetromino number $s(\lambda) \geq n / 2$ and $m-n / 2$ additional dominos, i.e., $d(\lambda) \geq n / 2+m$, then we can construct an $n^{\prime}$-vertex graph $G$ in polynomial time such that

$$
\operatorname{imm}_{\lambda}(G)=\underbrace{(-1)^{m-n / 2} \cdot 2^{n / 2} \cdot \chi_{\lambda}\left(\theta_{n / 2}\right)}_{=: c_{1}} \cdot \# \operatorname{PerfMatch}(H) .
$$

2. For any $k \leq \frac{s(\lambda)}{3}$ such that $d(\lambda) \geq m+n+2 k n-5 k$ : We can construct an $n^{\prime}$-vertex graph $G$ in polynomial time such that

$$
\operatorname{imm}_{\lambda}(G)=\underbrace{(-1)^{m+2 k n-3 k} \cdot(2 k) ! \cdot 2^{3 k} \cdot \chi_{\lambda}\left(\theta_{3 k}\right)}_{=: c_{2}} \cdot \# \operatorname{Match}(H, k) .
$$

Proof. For the first part, we define $G$ as follows:

1. Replace each edge $u v \in E(H)$ with a fresh copy $Q_{u v}$ of the edge gadget $Q$. Identify $u$ and $v$ with the endpoints of $Q$.

2. Add $d^{\prime}=d(\lambda)-(n / 2+m)$ padding digons and $z(\lambda)$ isolated padding vertices with self-loops.

By Lemma 27 we have $\operatorname{imm}_{\lambda}(G)=\operatorname{imm}_{\lambda}^{*}(G)$, where $\operatorname{imm}_{\lambda}^{*}(G)$ sums over the set $\mathcal{C}^{*}$ of consistent cycle covers. Any cycle cover $C \in \mathcal{C}^{*}$ includes all padding elements. Apart from padding elements, $C$ consists of the active states of some gadgets and the passive states of the remaining gadgets; let $M(C) \subseteq E(H)$ denote the set of pairs $u v$ such that $Q_{u v}$ is active in $C$. Since active gadget states must be vertex-disjoint, and all vertices of $G$ must be covered by cycles in $C$, the set $M(C)$ induces a perfect matching $M(C) \in \mathcal{M}_{n / 2}(H)$ in $H$. Conversely, given a perfect matching $M$ of $H$, let $\mathcal{C}_{M}^{*} \subseteq \mathcal{C}^{*}$ denote the set of consistent cycle covers with $M(C)=M$. By grouping the terms in $\operatorname{imm}_{\lambda}^{*}(G)$, we obtain

$$
\operatorname{imm}_{\lambda}^{*}(G)=\sum_{M \in \mathcal{M}_{n / 2}(H)} \underbrace{\sum_{C \in \mathcal{C}_{M}^{*}} \chi_{\lambda}(C) \cdot w(C)}_{=: a(M)} .
$$

To calculate $a(M)$, we investigate the set $\mathcal{C}_{M}^{*}$ : Each cycle cover $C \in \mathcal{C}_{M}^{*}$ is obtained by

- choosing an active state for each of the $n / 2$ gadgets $Q_{u v}$ with $u v \in M$, each inducing the weight $w(u v)$,

- adding the passive state (of weight -1 ) at the remaining gadgets, for a total weight of $(-1)^{m-n / 2}$, and

- adding all padding elements, all of weight 1.

The total weight is thus $(-1)^{m-n / 2}$. As choices can only be made at active states, the formats of cycle covers in $\mathcal{C}_{M}^{*}$ are given by the partition product

$$
\left\{\left(2^{2}\right),\left(2^{2}\right),(4),(4)\right\}^{\times n / 2} \times\left\{\left(2^{d(\lambda)-n}, 1^{z(\lambda)}\right)\right\} .
$$

Viewed as a multiset of partitions, this product amounts to $2^{n / 2}$ copies of $\theta_{n / 2}$ : After choosing one of the formats $\left\{\left(2^{2}\right),(4)\right\}$ for each of $n / 2$ entries, we can choose the first or second copy of this format. This shows that $a(M)=(-1)^{m-n / 2} \cdot 2^{n / 2} \cdot \chi_{\lambda}\left(\theta_{n / 2}\right)$ and thus proves (8).

For the second part, we construct the graph $G$ in a similar way, but we need to add some additional structures to account for the fact that most vertices are unmatched in a $k$-matching for $k \ll n$.

1. Replace all edges of $H$ by edge gadgets.

2. For each vertex $v \in V(H)$, add a switch vertex $s_{v}$ and connect it to $v$ with a switch digon. 
3. Add $2 k$ receptor vertices. Add a receptor edge between each pair of receptor and switch vertex, then replace these edges by edge gadgets.

4. Add $d^{\prime}=d(\lambda)-(m+n+2 k n-5 k)$ isolated digons and $z(\lambda)$ isolated vertices.

Any cycle cover $C \in \mathcal{C}^{*}$ then consists of the following cycles:

- Each receptor vertex must be covered by an active edge gadget. Then the other endpoint of that gadget is the switch vertex of some vertex in $H$. The remaining $2 k \cdot(n-1)$ edge gadgets incident with receptor vertices are passive. There are $(2 k)$ ! ways of matching the $2 k$ receptor vertices to $2 k$ fixed switch vertices with active gadgets.

- The $n-2 k$ switch vertices not touched by active gadgets from receptor vertices must be covered by switch digons.

- By the previous item, $2 k$ vertices in $H$ are left to be covered by $k$ active edge gadgets that represent edges in $H$. As active edge gadgets are vertex-disjoint, they induce a $k$-matching $M(C)$ in $H$, and they contribute weight $\prod_{u v \in M(C)} w(u v)$.

- Overall, there are $m-k+2 k \cdot(n-1)=m+2 k n-3 k$ passive edge gadgets in $C$, each contributing weight -1 . With padding digons and loops in $G$, there are $d(\lambda)-6 k$ digons and $z(\lambda)$ loops in $C$.

The third item describes how $C$ induces a $k$-matching $M(C) \in \mathcal{M}_{k}(H)$. Conversely, we can observe (as in the first case) that any $k$-matching $M$ of $H$ induces consistent cycle covers with a total contribution of

$$
(-1)^{m+2 k n-3 k} \cdot(2 k) ! \cdot 2^{3 k} \cdot w(M) \cdot \chi_{\lambda}\left(\theta_{3 k}\right) .
$$

Note that the factor $(2 k)$ ! stems from the different ways receptor vertices can match to switch vertices.

Remark. Why not use self-loops to handle the switch vertices of matched vertices? It seemed to work fine when dealing with staircases in Lemma 20? The reason is that doing so may require us to peel less than $d(\lambda)$ dominos from $\lambda$ to accommodate these self-loops. As a toy example for the complications that can arise this way, consider $\lambda=\left(3,1^{2}\right)$ with $d(\lambda)=2$ and $\rho=\left(2,1^{3}\right)$. There is only one domino in $\rho$, and we have $\chi_{\lambda}(\rho)=0$, since this single domino can be peeled as $\square$ or $\boxminus$, leading to a cancellation. If we peel all $d(\lambda)$ dominos from $\lambda$, then no such cancellations can occur, since Lemma 22 guarantees the same parity of the peeled domino tilings.

\subsection{Analyzing the character values}

In this section, we analyze $\chi_{\lambda}\left(\theta_{s}\right)$ for the partition product $\theta_{s}$ defined in (7). First, we collect a few facts on $F$ that can be checked by simple manual calculations. In the following, recall that a domino tiling of a $2 t$-box skew shape $\gamma$ is a border strip tableau of format $\left(2^{t}\right)$. We call it even/odd if its number of vertical $\boxminus$ pieces is even/odd. By Lemma 22, all domino tilings of $\gamma$ have the same parity. Also recall Section 3.3 for the required definitions related to partition products.

Fact 29. For $F=\left\{\left(2^{2}\right),(4)\right\}$, we have $\alpha_{F}(\gamma) \neq 0$ iff $\gamma$ is a non-vanishing tetromino. Furthermore, for any non-vanishing tetromino, the sign of $\alpha_{F}(\gamma)$ is given by the parity of its domino tilings, see Table 2:

$$
\alpha_{F}(\gamma)= \begin{cases}+2 & \gamma \text { has even domino tilings } \\ -2 & \gamma \text { has odd domino tilings }\end{cases}
$$

Proof. Let $\Gamma_{F}$ denote the skew shapes that admit a border strip tableau with a format from $F=\left\{\left(2^{2}\right),(4)\right\}$. We observe that every shape in $\Gamma_{F}$ admits a domino tiling with two dominos, and that $\Gamma_{F}$ therefore is a subset of the nine connected 4-box skew shapes from Table 1 and the four disconnected two-domino skew shapes. Furthermore: 


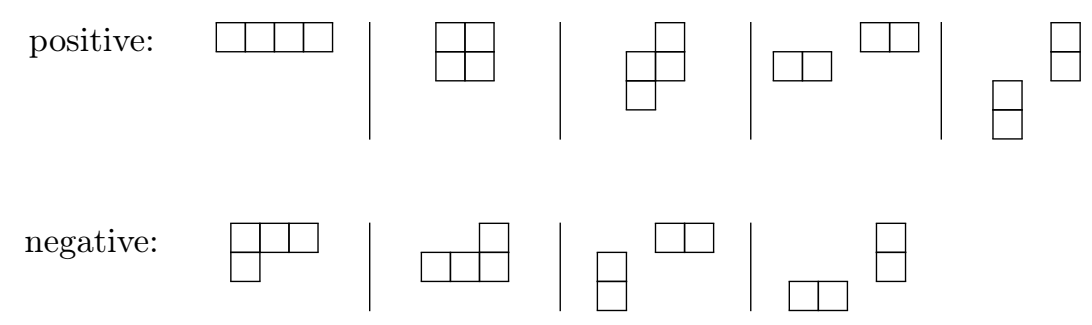

Table 2: The shapes $\gamma$ with $\alpha_{F}(\gamma) \neq 0$, grouped by sign.

- We have $\alpha_{F}(\gamma) \in\{-2,2\}$ if $\gamma$ consists of two disconnected dominos, and the sign is positive iff both dominos have the same orientation. Indeed, $\gamma$ admits exactly two border strip tableaux $T_{1}, T_{2}$ with formats from $F$, which are domino tilings with the same height sign.

- We have $\alpha_{F}(\boxplus)=2$, since the square admits two border strip tableaux with block formats from $F$. Both tableaux are domino tilings with the same height sign.

- The remaining connected 4-box shapes $\gamma$ are border strips. As such, they admit one border strip tableau of format $\left(2^{2}\right)$ and (4) each, implying that $\alpha_{F}(\gamma) \in\{-2,0,2\}$. We have $\alpha_{F}(\gamma) \neq 0$ iff the height of $\gamma$ and the unique domino tiling of $\gamma$ agree in parity. (Recall that the height ht $(\gamma)$ is the number of touched rows minus one.) The sign is positive iff the tiling is even.

These observations together prove the statement.

We can now prove that the relevant characters in the tetromino-based reduction do not vanish.

Lemma 30. For any partition $\lambda \vdash n^{\prime}$ and integer $s \leq s(\lambda)$, we have $\chi_{\lambda}\left(\theta_{s}\right) \neq 0$.

Proof. By Fact 29, we have $\gamma \in \Gamma_{F}$ and $\alpha_{F}(\gamma) \neq 0$ for a 4-box skew shape $\gamma$ iff $\gamma$ is a non-vanishing tetromino. Let $D=\{(2)\}$ and $S=\{(1)\}$; then $\Gamma_{D}=\{\square, \boxminus\}$ and $\Gamma_{S}=\{\square\}$. Define the set of skew shape tableaux

$$
\mathcal{S}=\mathcal{S}(\lambda, \underbrace{\Gamma_{F}, \ldots, \Gamma_{F}}_{s \text { times }}, \underbrace{\Gamma_{D}, \ldots, \Gamma_{D}}_{d(\lambda)-2 s \text { times }}, \underbrace{\Gamma_{S}, \ldots, \Gamma_{S}}_{z(\lambda) \text { times }})
$$

and let $t=d(\lambda)-s+z(\lambda)$ be the number of sets of skew shapes in the above list. For $i \in[t]$, let $\alpha_{i} \in\left\{\alpha_{F}, \alpha_{D}, \alpha_{S}\right\}$ be the coefficient function for the $i$-th set in the list. By Lemma 14, we have

$$
\chi_{\lambda}\left(\theta_{s}\right)=\sum_{\substack{S \in \mathcal{S} \text { with } \\ \text { shapes } \gamma_{1} \ldots \gamma_{t}}} \prod_{i=1}^{t} \alpha_{i}\left(\gamma_{i}\right) .
$$

It follows that every tableau $S \in \mathcal{S}$ with non-zero weight in the above sum peels $s$ non-vanishing tetrominos from $\lambda$, followed by $d(\lambda)-2 s$ dominos, and $z(\lambda)$ singleton boxes. The tetrominos and dominos tile $\lambda / \mu$, where $\mu$ is the staircase of $\lambda$.

Since $s \leq s(\lambda)$, at least one tableau $S \in \mathcal{S}$ exists, and we can prove the lemma by showing that all tableaux are counted with the same sign in (10). Towards this, note that any skew shape tableau $S \in \mathcal{S}$ can be turned into a border strip tableau $B(S)$ of $\lambda$ that peels $d(\lambda)$ dominos and $z(\lambda)$ singleton boxes from $\lambda$ : Simply tile each tetromino $\gamma$ in $S$ arbitrarily with two dominos. By Fact 29, we know that $\alpha_{F}(\gamma)$ is positive/negative iff the tiling of $\gamma$ is even/odd, so $\prod_{i=1}^{t} \alpha_{i}\left(\gamma_{i}\right)$ is positive/negative iff the domino tiling of $\lambda / \mu$ induced by $B(S)$ is even/odd. (Singleton boxes can be ignored, as they contribute the factor +1 .) But by Lemma 22, all domino tilings of $\lambda / \mu$ have the same parity. Therefore, all terms in (10) have the same sign.

Corollary 31. The coefficients $c_{1}$ and $c_{2}$ defined in Lemma 28 are both non-zero. 


\subsection{Reductions}

As in Section 5.3, we collect the previous arguments to obtain a reduction from counting matchings to immanants for partitions with large non-vanishing tetromino number.

Lemma 32. The following can be achieved in polynomial time and with polynomial-sized arithmetic circuits:

1. Given an n-vertex graph $H$ of maximum degree 3 and a partition $\lambda$ with $s(\lambda) \geq n / 2$ and $d(\lambda) \geq 2 n$, compute a digraph $G$ and a number $c \in \mathbb{Q}$ such that \#PerfMatch $(H)=c \cdot \operatorname{imm}_{\lambda}(G)$.

2. Given an n-vertex graph $H$ and $k \in \mathbb{N}$, and a partition $\lambda$ with $s(\lambda) \geq 3 k$ and $d(\lambda) \geq n^{2}+n+2 k n-5 k$, compute a digraph $G$ and a number $c \in \mathbb{Q}$ such that $\# \operatorname{Match}(H, k)=c \cdot \operatorname{imm}_{\lambda}(G)$.

Proof. For the first part, let $H$ be a graph with $n$ vertices and maximum degree 3 , so that $m \leq \frac{3}{2} n$. Note that $d(\lambda) \geq n+m$ by assumption. We construct a graph $G$ via Lemma 28 such that $\operatorname{imm}_{\lambda}(G)=c_{1} \cdot \# \operatorname{PerfMatch}(H)$. For the second part, we can use Lemma 28 to construct a graph $G$ such that $\operatorname{imm}_{\lambda}(G)=c_{2} \cdot \# \operatorname{Match}(H, k)$. We have $c_{1}, c_{2} \neq 0$ by Corollary 31 .

\section{Completing the proofs}

Let $\Lambda$ be a family of partitions with unbounded $b(\Lambda)$. We compose the constructions from the preceding sections to an overall hardness proof for $\operatorname{Imm}(\Lambda)$. This requires us to find sequences of partitions within $\Lambda$ that are dense enough and supply sufficiently many boxes to the right of the first column. In the sub-polynomial growth regime, we also need to ensure a sufficiently large supply of dominos; this can be achieved by having a large number of boxes in the first column.

Definition 33. Given a polynomial-time computable function $g: \mathbb{N} \rightarrow \mathbb{N}$, a family of partitions $\Lambda$ supports growth $g$ if there is a sequence $\Lambda^{\prime}=\left(\lambda^{(1)}, \lambda^{(2)}, \ldots\right)$ in $\Lambda$ such that $\lambda^{(n)}$ satisfies $b\left(\lambda^{(n)}\right) \geq g(n)$ and $\left\|\lambda^{(n)}\right\|=$ $\Theta(n)$. We also say that $\Lambda$ supports growth $g$ via $\Lambda^{\prime}$. We say that $\Lambda$ computationally supports growth $g$ if $\lambda^{(n)}$ can be computed in polynomial time from $n$.

We are ready to prove the main theorems of this paper. To this end, we distinguish whether $\Lambda$ supports polynomial growth (for a reduction from \#PerfMatch) or only sub-polynomial growth (for a reduction from \#Match ${ }^{(g)}$ for any growth $g$ supported by $\Lambda$ ). Recall that, by known algorithms [23, 8], we have $\operatorname{Imm}(\Lambda) \in \mathrm{FP}$ and $\operatorname{Imm}(\Lambda) \in \mathrm{VP}$ for any family $\Lambda$ with $b(\Lambda)<\infty$.

Proof of Theorem 1. The tractability part is known. For hardness, we reduce from \#PerfMatch: Let $H$ be an $n$-vertex bipartite graph for which we want to compute \#PerfMatch $(H)$. Let $\alpha>0$ be maximal such that $\Lambda$ supports growth $\Omega\left(n^{\alpha}\right)$. We find a partition $\lambda \in \Lambda$ with $\|\lambda\|=\Theta\left(n^{1 / \alpha}\right)$ such that $b(\lambda) \geq 20 n$. Then Lemma 18 guarantees that at least one of $s(\lambda) \geq 3.5 n$ or $w(\lambda) \geq \sqrt{20 n}-1$ holds. In either case, using the first cases of Lemmas 26 and 32, we compute a graph $G$ on $\|\lambda\|$ vertices and a number $c \in \mathbb{Q}$ such that \#PerfMatch $(H)=c \cdot \operatorname{imm}_{\lambda}(G)$ holds. Overall, this yields polynomial-time and c-reductions from \#PerfMatch to $\operatorname{Imm}(\Lambda)$, showing \#P-hardness and VNP-completeness of the latter. The lower bound under \#ETH follows since $\|\lambda\|=\Theta\left(n^{1 / \alpha}\right)$.

If $\Lambda$ supports only sub-polynomial growth, the proof proceeds similarly. In this case, we can find a sequence of partitions in which most rows have width 1 , which allows us to peel a large amount of dominos from the left-most column.

Proof of Theorem 2. If $\Lambda$ supports polynomial growth, we use Theorem 1. Otherwise, let $g \in \omega(1)$ be a growth supported by $\Lambda$. We reduce from $\# \operatorname{Match}^{(h)}$ with $h(n)=\sqrt{g(n)} / 24$ : Let $(H, k)$ be an instance for \#Match ${ }^{(h)}$ with an $n$-vertex graph $H$ and $k \leq h(n)$. Using the growth condition on $\Lambda$ and $g \in O\left(n^{0.1}\right)$, we find a partition $\lambda \in \Lambda$ with $b(\lambda) \geq 24 k$ and at least $2 n^{2}+b(\lambda)$ boxes in the first column, which implies $d(\lambda) \geq n^{2}$. With the bound on $b(\lambda)$, Lemma 18 yields that $s(\lambda) \geq 3 k$ or $w(\lambda) \geq \sqrt{24 k}-1$. In either case, 
using the second case of Lemmas 26 and 32 , we compute a graph $G$ on $\|\lambda\|$ vertices and a number $c \in \mathbb{Q}$ such that \#Match $(H, k)=c \cdot\left[x^{t}\right] \operatorname{imm}_{\lambda}(G)$, where $t=2 k$ for the staircase-based reduction (Lemma 26) and $t=0$ for the tetromino-based reduction (Lemma 32 ).

Note that we can compute $\left[x^{t}\right] \operatorname{imm}_{\lambda}(G)$ via polynomial interpolation from the values $\operatorname{imm}_{\lambda}\left(G_{i}\right)$ for $i \in\{0, \ldots,\|\lambda\|\}$, where $G_{i}$ is the graph obtained from $G$ by replacing each edge of weight $x$ with an edge of weight $i$. Overall, we obtain a polynomial-time Turing reduction from \# Match ${ }^{(h)}$ to $\operatorname{Imm}(\Lambda)$, which implies by Lemma 4 that $\operatorname{Imm}(\Lambda) \notin \mathrm{FP}$ unless FPT $=\# \mathrm{~W}[1]$. An analogous statement holds in the algebraic setting: As polynomial interpolation amounts to solving a system of linear equations, which can be performed with polynomial-sized circuits, we obtain a parameterized c-reduction from the p-family \#Match ${ }^{(h)}$ to the p-family $\operatorname{Imm}\left(\Lambda^{\prime}\right)$.

\section{Conclusion and future work}

We established a dichotomy for the complexity of immanants, concluding a sequence of partial results obtained throughout the last four decades $[23,9,7,27,20]$.

Let us note that immanants are not the only way of generalizing permanents and determinants into a family of matrix forms. Other examples include the cover polynomials [14, 5] and the fermionants [27, 2], which are also sum-products over row-column permutations of a matrix. Unlike the immanant however, these families do not exhibit gradual progressions from easy to hard cases, and they feature no non-trivial easy cases beside the determinant.

Our result for immanants also prompts several interesting follow-up questions.

Modular immanants. Writing $\lambda^{\prime}$ for the transpose of a partition $\lambda$, it is known that $\chi_{\lambda}$ and $\chi_{\lambda^{\prime}}$ are equivalent modulo 2. This renders $\lambda$-immanants tractable over $\mathbb{Z}_{2}$ for partitions $\lambda$ with constantly many boxes outside of the first column or row. Are these the only tractable immanants over $\mathbb{Z}_{2}$ ? Which immanants are tractable over $\mathbb{Z}_{p}$ for odd primes $p$ ? These questions lead into the tricky territory of representation theory over fields of positive characteristic.

Planar graphs. The permanent is polynomial-time solvable for bi-adjacency matrices of planar bipartite graphs. This is a consequence of the classical FKT algorithm [35], which expresses the number of perfect matchings in a planar graph $G$ as a determinant. The relevant matrix is derived from the adjacency matrix of $G$ by flipping signs according to a Pfaffian orientation of $G$. Can this algorithmic idea be generalized to more general immanants on bi-adjacency matrices of planar bipartite graphs? Which immanants remain hard on planar graphs?

Removing weights. Our proof establishes hardness for matrices with general entries from $\mathbb{Z}$ : Even though the source problems \#PerfMatch and \#Match are hard for unweighted graphs, the edge-gadget and interpolation steps introduce non-unit weights. It may however still be possible to establish hardness for 0-1 matrices, as known for the permanent. While gadgets for simulating edge-weights are known, they change the formats of the relevant cycle covers, and it is more difficult to argue about character values on these formats.

\section{Acknowledgments}

I thank Christian Engels for introducing me to the immanants and Nathan Lindzey for introducing me to the representation theory of $S_{n}$. 


\section{References}

[1] A. I. Barvinok. Computational complexity of immanants and representations of the full linear group. Functional Analysis and Its Applications, 24:144-145, 1990.

[2] Andreas Björklund, Petteri Kaski, and Ryan Williams. Generalized Kakeya sets for polynomial evaluation and faster computation of fermionants. Algorithmica, 81(10):4010-4028, 2019.

[3] Markus Bläser and Radu Curticapean. The complexity of the cover polynomials for planar graphs of bounded degree. In Filip Murlak and Piotr Sankowski, editors, Mathematical Foundations of Computer Science 2011 - 36th International Symposium, MFCS 2011, Warsaw, Poland, August 22-26, 2011. Proceedings, volume 6907 of Lecture Notes in Computer Science, pages 96-107. Springer, 2011.

[4] Markus Bläser and Radu Curticapean. Weighted counting of $k$-matchings is \#W[1]-hard. In Dimitrios M. Thilikos and Gerhard J. Woeginger, editors, Parameterized and Exact Computation - 7th International Symposium, IPEC 2012, Ljubljana, Slovenia, September 12-14, 2012. Proceedings, volume 7535 of Lecture Notes in Computer Science, pages 171-181. Springer, 2012.

[5] Markus Bläser, Holger Dell, and Mahmoud Fouz. Complexity and approximability of the cover polynomial. Comput. Complex., 21(3):359-419, 2012.

[6] Markus Bläser and Christian Engels. Parameterized Valiant's classes. In Bart M. P. Jansen and Jan Arne Telle, editors, 14th International Symposium on Parameterized and Exact Computation, IPEC 2019, September 11-13, 2019, Munich, Germany, volume 148 of LIPIcs, pages 3:1-3:14. Schloss Dagstuhl Leibniz-Zentrum für Informatik, 2019.

[7] Jean-Luc Brylinski and Ranee Brylinski. Complexity and completeness of immanants. CoRR, cs.CC/0301024, 2003.

[8] Peter Bürgisser. Completeness and Reduction in Algebraic Complexity Theory, volume 7 of Algorithms and computation in mathematics. Springer, 2000.

[9] Peter Bürgisser. The computational complexity of immanants. SIAM J. Comput., 30(3):1023-1040, 2000.

[10] Peter Bürgisser. The computational complexity to evaluate representations of general linear groups. SIAM J. Comput., 30(3):1010-1022, 2000.

[11] Peter Bürgisser, Michael Clausen, and Mohammad Amin Shokrollahi. Algebraic complexity theory, volume 315 of Grundlehren der mathematischen Wissenschaften. Springer, 1997.

[12] Jin-yi Cai, Xi Chen, and Dong Li. A quadratic lower bound for the permanent and determinant problem over any characteristic $\neq 2$. In Cynthia Dwork, editor, Proceedings of the 40th Annual ACM Symposium on Theory of Computing, Victoria, British Columbia, Canada, May 17-20, 2008, pages 491-498. ACM, 2008.

[13] Gordon G. Cash. Immanants and immanantal polynomials of chemical graphs. J. Chem. Inf. Comput. Sci., 43(6):1942-1946, 2003.

[14] Fan R. K. Chung and Ronald L. Graham. On the cover polynomial of a digraph. J. Comb. Theory, Ser. $B, 65(2): 273-290,1995$.

[15] Radu Curticapean. Counting matchings of size $k$ is \#w[1]-hard. In Fedor V. Fomin, Rusins Freivalds, Marta Z. Kwiatkowska, and David Peleg, editors, Automata, Languages, and Programming - 40th International Colloquium, ICALP 2013, Riga, Latvia, July 8-12, 2013, Proceedings, Part I, volume 7965 of Lecture Notes in Computer Science, pages 352-363. Springer, 2013. 
[16] Radu Curticapean. Block interpolation: A framework for tight exponential-time counting complexity. In Magnús M. Halldórsson, Kazuo Iwama, Naoki Kobayashi, and Bettina Speckmann, editors, Automata, Languages, and Programming - 42nd International Colloquium, ICALP 2015, Kyoto, Japan, July 6-10, 2015, Proceedings, Part I, volume 9134 of Lecture Notes in Computer Science, pages 380-392. Springer, 2015.

[17] Radu Curticapean. The simple, little and slow things count: on parameterized counting complexity. $\mathrm{PhD}$ thesis, Saarland University, 2015.

[18] Radu Curticapean, Holger Dell, and Dániel Marx. Homomorphisms are a good basis for counting small subgraphs. In Hamed Hatami, Pierre McKenzie, and Valerie King, editors, Proceedings of the 49th Annual ACM SIGACT Symposium on Theory of Computing, STOC 2017, Montreal, QC, Canada, June 19-23, 2017, pages 210-223. ACM, 2017.

[19] Radu Curticapean and Dániel Marx. Complexity of counting subgraphs: Only the boundedness of the vertex-cover number counts. In 55th IEEE Annual Symposium on Foundations of Computer Science, FOCS 2014, Philadelphia, PA, USA, October 18-21, 2014, pages 130-139. IEEE Computer Society, 2014.

[20] Nicolas de Rugy-Altherre. Determinant versus permanent: Salvation via generalization? In Paola Bonizzoni, Vasco Brattka, and Benedikt Löwe, editors, The Nature of Computation. Logic, Algorithms, Applications - 9th Conference on Computability in Europe, CiE 2013, Milan, Italy, July 1-5, 2013. Proceedings, volume 7921 of Lecture Notes in Computer Science, pages 87-96. Springer, 2013.

[21] Jörg Flum and Martin Grohe. The parameterized complexity of counting problems. SIAM J. Comput., 33(4):892-922, 2004.

[22] W. Fulton, W.F.J. Harris, and J. Harris. Representation Theory: A First Course. Graduate Texts in Mathematics. Springer New York, 1991.

[23] W. Hartmann. On the complexity of immanants. Linear and Multilinear Algebra, 18(2):127-140, 1985.

[24] D. E. Littlewood and A. R. Richardson. Group characters and algebra. Philosophical Transactions of the Royal Society of London. Series A, Containing Papers of a Mathematical or Physical Character, 233:99-141, 1934.

[25] M. Lorenz. A Tour of Representation Theory. Graduate studies in mathematics. American Mathematical Society, 2018.

[26] I.G. Macdonald. Symmetric Functions and Hall Polynomials. Oxford classic texts in the physical sciences. Clarendon Press, 1998.

[27] Stephan Mertens and Cristopher Moore. The complexity of the fermionant and immanants of constant width [note]. Theory Comput., 9:273-282, 2013.

[28] T. Mignon and N. Ressayre. A quadratic bound for the determinant and permanent problem. International Mathematics Research Notices, 2004(79):4241-4253, 2004.

[29] Brendon Rhoades and Mark Skandera. Kazhdan-Lusztig immanants and products of matrix minors. Journal of Algebra, 304(2):793-811, 2006.

[30] B.E. Sagan. The Symmetric Group: Representations, Combinatorial Algorithms, and Symmetric Functions. Graduate Texts in Mathematics. Springer New York, 2013.

[31] I. Schur. Über eine Klasse von Matrizen, die sich einer gegebenen Matrix zuordnen lassen. PhD thesis, Friedrich-Wilhelms-Universität zu Berlin, 1901.

[32] I. Schur. Über endliche Gruppen und Hermitesche Formen. Mathematische Zeitschrift, 1:184-207, 1918. 
[33] Valery S. Shchesnovich. The permanent-on-top conjecture is false. Linear Algebra and its Applications, 490:196 - 201, 2016.

[34] Si-Hui Tan, Yvonne Gao, Hubert Guise, and Barry Sanders. SU(3) quantum interferometry with single-photon input pulses. Physical Review Letters, 110, 082012.

[35] H. N. V. Temperley and Michael E. Fisher. Dimer problem in statistical mechanics - an exact result. The Philosophical Magazine: A Journal of Theoretical Experimental and Applied Physics, 6(68):1061-1063, 1961.

[36] Leslie G. Valiant. Completeness classes in algebra. In Michael J. Fischer, Richard A. DeMillo, Nancy A. Lynch, Walter A. Burkhard, and Alfred V. Aho, editors, Proceedings of the 11h Annual ACM Symposium on Theory of Computing, April 30 - May 2, 1979, Atlanta, Georgia, USA, pages 249-261. ACM, 1979.

[37] Leslie G. Valiant. The complexity of computing the permanent. Theor. Comput. Sci., 8:189-201, 1979. 